\title{
Resonances over a potential well in an island
}

\author{
Johannes Sjöstrand and Maher Zerzeri
}

\begin{abstract}
In this paper we study the distribution of scattering resonances for a multidimensional semi-classical Schrödinger operator, associated to a potential well in an island at energies close to the maximal one that limits the separation of the well and the surrounding sea.

Résumé. Dans cet article, nous étudions la distribution des résonances pour l'opérateur de Schrödinger semi-classique multidimensionnel, associé à un puits de potentiel dans l'île aux énergies proche de celle qui délimite la séparation du puits et de la mer environnante.
\end{abstract}

\section{Introduction and main result}

In this work we consider resonances for a semi-classical Schrödinger operator with potential $V \in C^{\infty}\left(\mathbb{R}^{n} ; \mathbb{R}\right)$ (cf. (1.5)), where we assume that $n \geq 2$ and that

$$
\left\{\begin{array}{l}
V \text { has a holomorphic extension to a truncated } \\
\text { sector } \Gamma_{C}:=\left\{x \in \mathbb{C}^{n} ;|\Re x|>C,|\Im x|<\frac{1}{C}|\Re x|\right\} .
\end{array}\right.
$$

Here $C$ is some positive constant. We let $V$ also denote the extension. Assume

$$
V \longrightarrow 0, \quad \text { when } x \longrightarrow \infty \text { in } \Gamma_{C} \text {. }
$$

Let $E_{0}>0$. Assume that $V^{-1}(]-\infty, E_{0}[)=\mathscr{U}_{E_{0}} \sqcup \mathscr{S}_{E_{0}}$, where $\mathscr{U}_{E_{0}}, \mathscr{S}_{E_{0}}$ are open connected and mutually disjoint. We let $\mathscr{U}_{E_{0}}$ (the potential well) be the bounded component and $\mathscr{S}_{E_{0}}$ (the sea) be the unbounded one. When

$$
\overline{\mathscr{U}}_{E_{0}} \cap \overline{\mathscr{S}}_{E_{0}}=\varnothing
$$

Key words and phrases: resonances, semi-classical asymptotic, microlocal analysis, Schrödinger operator, potential well, maximal energy.

2010 Mathematics Subject Classification: primary 35J10; secondary 35B34, 35P20, $47 \mathrm{~A} 55$. 
the situation is quite well understood (see [11] and also [2]): Let

$$
p(x, \xi)=\xi^{2}+V(x)
$$

and assume that

$$
\begin{gathered}
V \text { is analytic in a neighborhood of } \overline{\mathscr{S}}_{E_{0}}, \\
H_{p}=\partial_{\xi} p \cdot \partial_{x}-\partial_{x} p \cdot \partial_{\xi} \text { has no trapped trajectories in } p^{-1}\left(E_{0}\right)_{\mid \overline{\mathscr{S}}_{E_{0}}} .
\end{gathered}
$$

Here a trapped trajectory is by definition a maximally extended integral curve of $H_{p}$ which is contained in some bounded set.

By suitably modifying the potential near $\overline{\mathscr{S}}_{E_{0}}$, we get a new potential $V^{\text {int }}$ which is equal to $V$ in a neighborhood of $\overline{\mathscr{U}}_{E_{0}}$ and $\geq E_{0}+\frac{1}{\mathcal{O}(1)}\left({ }^{1}\right)$ away from that neighborhood, so that $P^{\text {int }}=-h^{2} \Delta+V^{\text {int }}$ is self-adjoint with purely discrete spectrum in $]-\infty, E_{0}+\frac{1}{\mathcal{O}(1)}[$. The eigenvalues are distributed according to the semiclassical Weyl law and it was established in [2, Theorem 4 in Section IV or Theorem 2 in Section V] and [11, Proposition 9.6 and Theorem 9.7] that the resonances of

$$
P=-h^{2} \Delta+V
$$

in neigh $\left(E_{0}, \mathbb{C}\right)\left({ }^{2}\right)$ are related to the eigenvalues of $P^{\text {int }}$ in neigh $\left(E_{0}, \mathbb{R}\right)$ via a bijection

$$
b: \sigma\left(P^{\text {int }}\right) \cap \operatorname{neigh}\left(E_{0}, \mathbb{R}\right) \longrightarrow \operatorname{Res}(P) \cap \operatorname{neigh}\left(E_{0}, \mathbb{C}\right),
$$

such that $b(\mu)-\mu=\mathcal{O}(1) e^{-\frac{1}{\mathcal{O}(h)}}$. Here $\sigma\left(P^{\text {int }}\right)$ denotes the spectrum of $P^{\text {int }}$ and $\operatorname{Res}(P)$ the set of resonances, where both the eigenvalues and the resonances are counted with their natural multiplicity. See [7], [12], [13] and [14] for related results for potentials that may be non-analytic on any bounded set.

When increasing the energy level, we may have

$$
V^{-1}(]-\infty, E[)=\mathscr{U}_{E} \sqcup \mathscr{S}_{E}
$$

for $E-E_{0}>0$ small, where $\mathscr{U}_{E} \supset \mathscr{U}_{E_{0}}, \mathscr{S}_{E} \supset \mathscr{S}_{E_{0}}$ remain connected and disjoint until we reach a new energy $E_{0}=E_{0}^{\text {new }}$, for which (1.3) no longer holds. We typically may have

$$
\overline{\mathscr{U}}_{E_{0}} \cap \overline{\mathscr{S}}_{E_{0}}=\left\{x_{0}\right\},
$$

$\left({ }^{1}\right)$ Here we follow the convention that the expression "OOO(1)" in a denominator denotes a bounded positive quantity.

$\left({ }^{2}\right)$ Let $M$ be a topological space. Let $N$ be a subset of $M$. The set neigh $(N, M)$ denotes some neighborhood of $N$ in $M$. 


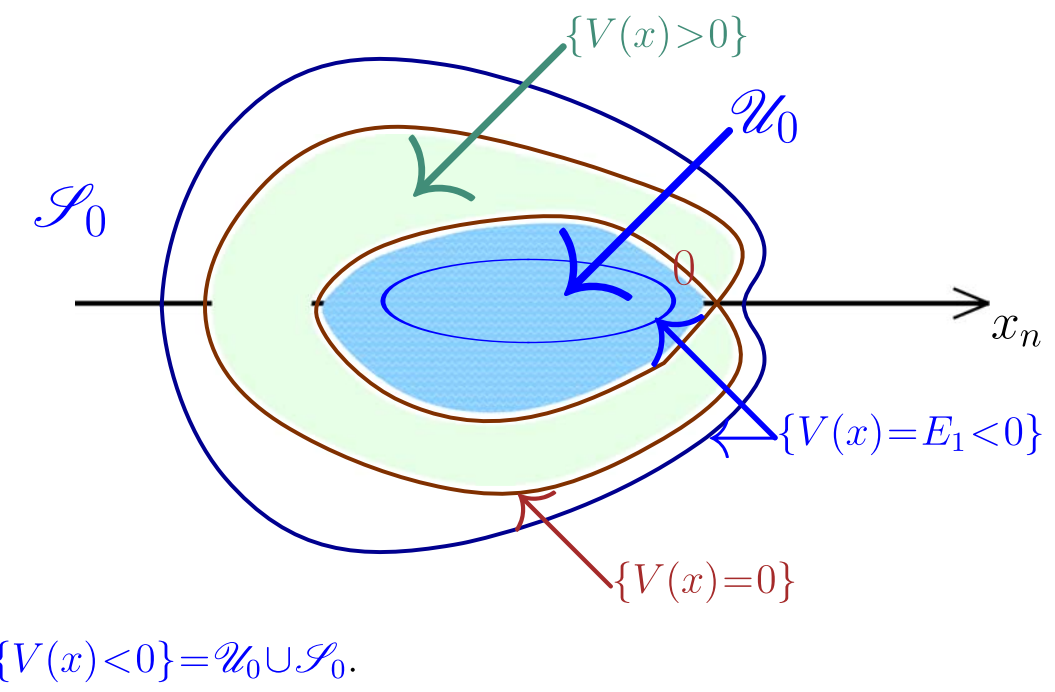

Figure 1. View from above. The topographic situation.

for some point $x_{0} \in \mathbb{R}^{n}$ while the other assumptions remain valid. In this work we study the distribution of resonances near the new energy level $E_{0}>0$.

For simplicity we now replace $V$ by $V-E_{0}$ (so that the energy level $E_{0}$ transforms to the level 0 ) and formulate our assumptions for the new potential $V \in$ $C^{\infty}\left(\mathbb{R}^{n} ; \mathbb{R}\right),(n>1)$ assumed to satisfy (1.1). Instead of (1.2) we assume

$$
V \longrightarrow-E_{0}, \quad \text { when } x \longrightarrow \infty \text { in } \Gamma_{C} \text {. }
$$

Assume that

$$
V^{-1}(]-\infty, 0[)=\mathscr{U}_{0} \sqcup \mathscr{S}_{0},
$$

where $\mathscr{U}_{0}, \mathscr{S}_{0}$ are open connected (and mutually disjoint). We let $\mathscr{U}_{0}$ (the potential well) be the bounded component and $\mathscr{S}_{0}$ (the sea) be the unbounded one. Define $p(x, \xi)$ as in (1.4). Assume that

$$
V \text { is analytic in a neighborhood of } \overline{\mathscr{S}}_{0} \text {. }
$$

Assume that

$$
\overline{\mathscr{U}}_{0} \cap \overline{\mathscr{S}}_{0}=\left\{x_{0}\right\}
$$

for some point $x_{0} \in \mathbb{R}^{n}$. After a translation, we may assume that $x_{0}=0$. 
We make the generic assumption that

$$
\left\{\begin{array}{l}
x_{0}=0 \text { is a non-degenerate critical } \\
\text { point for } V \text { of signature }(n-1,1) .
\end{array}\right.
$$

The point $(0,0) \in \mathbb{R}^{2 n}$ is a stationary point and hence a trapped trajectory for the Hamilton flow of $p$. Assume that

$$
\left\{\begin{array}{l}
\{(0,0)\} \text { is the only trapped trajectory } \\
\text { for the } H_{p} \text {-flow in }\left.p^{-1}(0)\right|_{\mathscr{S}_{0}} .
\end{array}\right.
$$

We have $V(0)=0$. Assume for simplicity that

$$
d V(x) \neq 0, \quad \text { when } x \in \partial \mathscr{U}_{0} \backslash\{0\} .
$$

As in [11] one can define a reference operator $P^{\text {int }}$ by increasing $V$ in $\mathscr{S}_{0}$ to get a potential $V^{\text {int }}$ which is $\geq 0$ away from $\mathscr{U}_{0}$. (Take for instance $V^{\text {int }}=1_{\mathbb{R}^{n} \backslash \mathscr{S}_{0}} V+1_{\mathscr{S}_{0}}$.) Then we have the standard Weyl asymptotics for the number of eigenvalues of $P^{\text {int }}$ in $]-\infty, E]$ when

$$
-\frac{1}{C} \leq E \leq-\delta
$$

for every fixed $0<\delta \ll 1$ and $C \gg 1$, stating that

$$
\left.\left.\left.\left.\#\left(\sigma\left(P^{\mathrm{int}}\right) \cap\right]-\infty, E\right]\right)=\frac{1}{(2 \pi h)^{n}}\left(\operatorname{vol}\left(p^{-1}(]-\infty, E\right]\right)_{\left.\right|_{\mathscr{U}_{0}}}\right)+o(1)\right)
$$

as $h \longrightarrow 0$ and uniformly for $E$ as in $(1.14) \cdot\left({ }^{3}\right)$

For $E \leq 0$, put

$$
\left.\left.\omega(E)=\operatorname{vol}\left(p^{-1}(]-\infty, E\right]\right)_{\left.\right|_{\mathscr{U}_{0}}}\right)
$$

so that

$$
\omega(E)=C_{n} \int_{\mathscr{U}_{0}}(E-V(x))_{+}^{\frac{n}{2}} d x
$$

where $C_{n}=\operatorname{vol}\left(B_{\mathbb{R}^{n}}(0,1)\right)=\frac{\pi^{\frac{n}{2}}}{\Gamma\left(\frac{n}{2}+1\right)}$. Since $n \geq 2$, we see that $\omega \in C^{1}\left(\left[-\frac{1}{C}, 0\right]\right)$ and that

$$
\omega^{\prime}(E)=\frac{\pi^{\frac{n}{2}}}{\Gamma\left(\frac{n}{2}\right)} \int_{\mathscr{U}_{0}}(E-V(x))_{+}^{\frac{n}{2}-1} d x .
$$

Let $\omega$ also denote a $C^{1}$-extension to the interval $\left[-\frac{1}{C}, \frac{1}{C}\right]$ so that (1.16) holds for $E \leq 0$ and so that $\omega(E)$ is well-defined up to a term $o(E)$ for $0 \leq E \leq \frac{1}{C}$.

$\left(^{3}\right)$ Here for $A \subset T^{*} \mathbb{R}^{n}, B \subset \mathbb{R}^{n}$ we write $A_{\mid B}=A \cap \pi_{x}^{-1}(B)$ where $\pi_{x}: T^{*} \mathbb{R}^{n} \longrightarrow \mathbb{R}^{n}$ is the canonical projection, given by $\pi_{x}(x, \xi)=x$. 
Theorem 1.1. Let $V \in C^{\infty}\left(\mathbb{R}^{n} ; \mathbb{R}\right)$ and define $P, p$ as in (1.5), (1.4). Let $E_{0}>0$ and assume (1.1), (1.7), (1.8), (1.9), (1.10), (1.11), (1.12) and (1.13). Let $\omega(E)$ be a $C^{1}$ function on $\left[-\frac{1}{C}, \frac{1}{C}\right]$ satisfying (1.16) for $E \leq 0$.

Let $C_{0}>0$. Then for every $0<\delta \leq \frac{1}{2}$, there exists $0<\varepsilon(\delta) \ll 1$ such that for every $0<\varepsilon \leq \varepsilon(\delta)$ and $0<h \leq h(\delta, \varepsilon)$ small enough:

(A) The number of resonances (of $P$ ) in $]-C_{0} \varepsilon, \varepsilon[+i]-\varepsilon,-\delta \varepsilon\left[\right.$ is $\mathcal{O}_{\delta}\left(h^{-n} \varepsilon^{n}\right)$.

(B) For all $a, b \in]-C_{0} \varepsilon, \varepsilon[$ with $a<b$, the number of resonances in $] a, b[+$ $i]-\delta \varepsilon, 0]$ is equal to $(2 \pi h)^{-n}(\omega(b)-\omega(a)+\mathcal{O}(\delta|\ln \delta| \varepsilon))$, uniformly with respect to $a, b, h$.

More precise results are known when $n=1$. In this case the function $\omega$ has a logarithmic singularity at zero. See [1] and [8].

At least formally our result is similar to recent ones about Helmholtz resonators and other capting devices. See [5], [15] and also [3].

The remainder of the paper is devoted to the proof of Theorem 1.1. We shall use suitable escape functions and the corresponding spaces of distributions with exponential phase space weights as developed in [11]. The main work will take place near the island and we found it convenient to use the global framework of [11]. The control near infinity could also be obtained using complex distorsion techniques. (See $[6]$ for an overview.)

Acknowledgments. We are grateful to the referee for useful remarks that have led to improvements of the exposition.

The IMB receives support from the EIPHI Graduate School (contract ANR-17-EURE-0002).

\section{Outline}

\subsection{Escape functions (Sections 3,4 )}

After a linear change of coordinates, we may assume that near $x=\left(x^{\prime}, x_{n}\right)=0$

$$
p(x, \xi)=\underbrace{\frac{\varkappa}{2}\left(\xi_{n}^{2}-x_{n}^{2}\right)+q\left(x^{\prime}, \xi^{\prime}\right)}_{=: p_{0}(x, \xi)}+\mathcal{O}\left(|x|^{3}\right),
$$

where $\varkappa>0$ and $q$ is a positive definite quadratic form. Moreover, $x_{n}<0$ in the well and $x_{n}>0$ in the sea. Assume for simplicity that $\varkappa=1$.

Let $G_{0}(x, \xi)=x_{n} \xi_{n}$. Then $H_{p_{0}} G_{0}=x_{n}^{2}+\xi_{n}^{2}$. We will define $G=G^{\varepsilon}$ near $(0,0)$ as a truncation of $G_{0}$. See (3.5). We show that, with $\rho=(x, \xi)$ :

$$
\partial_{\rho}^{\alpha} G(\rho)=\mathcal{O}(1)\left(\varepsilon+\rho^{2}\right)^{1-\frac{|\alpha|}{2}}, \quad \alpha \in \mathbb{N}^{2 n}
$$


and that there exists $C \geq 1$ such that in neigh $\left(0, \mathbb{R}^{2 n}\right)$ :

$$
\text { If } p(\rho)<-\frac{\varepsilon}{C}+\frac{\rho^{2}}{C} \quad \text { and } \quad x_{n}(\rho) \geq 0, \quad \text { then } H_{p} G \asymp \varepsilon+\rho^{2} \text {. }
$$

Here we write $X \asymp Y$ for $X, Y \in \mathbb{R}$ if $X, Y$ have the same sign (or vanish) and $X=$ $\mathcal{O}(Y)$ and $Y=\mathcal{O}(X)$.

We add a bump at the saddle point in order to create a barrier between the well and the sea: Let $\chi(x, \xi)=e^{-(\beta x)^{2}-(\beta \xi)^{2}}$ where $\beta>0$ is small but fixed. Put

$$
p_{\varepsilon}=p+\chi_{\varepsilon}, \quad \chi_{\varepsilon}(x, \xi)=\varepsilon \chi\left(\frac{x}{\sqrt{\varepsilon}}, \frac{\xi}{\sqrt{\varepsilon}}\right) .
$$

Then there exist $b, c>0$ such that:

$$
\text { If } p_{\varepsilon}(\rho)<b \varepsilon+c \rho^{2} \quad \text { and } \quad x_{n}(\rho) \geq 0, \quad \text { then } H_{p_{\varepsilon}} G \asymp \varepsilon+\rho^{2} \text {. }
$$

Proposition 2.1. Possibly after a dilation in $\varepsilon$ in the definition of $G$ we have for $0<t \ll 1$ and all $\rho$ in neigh $\left(0, \mathbb{R}^{2 n}\right)$ with $x_{n}(\rho) \geq 0$ :

$$
\begin{aligned}
& \text { If } \Re p\left(\rho+i t H_{G}(\rho)\right) \leq-\frac{\varepsilon}{\widetilde{C}}+\frac{\rho^{2}}{2 C}, \quad \text { then } \Im p\left(\rho+i t H_{G}(\rho)\right) \asymp-t\left(\varepsilon+\rho^{2}\right) . \\
& \text { If } \Re p_{\varepsilon}\left(\rho+i t H_{G}(\rho)\right) \leq b \varepsilon+\frac{c}{2} \rho^{2}, \quad \text { then } \Im p_{\varepsilon}\left(\rho+i t H_{G}(\rho)\right) \asymp-t\left(\varepsilon+\rho^{2}\right) .
\end{aligned}
$$

\subsection{Resolvents (Sections 5, 6, 7, 8, 9, 10, A)}

Let $P_{\varepsilon}$ be the $h$-Weyl quantization of $p_{\varepsilon}$. Let $t>0$ be small enough and fixed. We can extend $G$ as a classical escape function ([9] and [11]) to $\mathbb{R}_{x}^{n} \times \mathbb{R}_{\xi}^{n}$, equal to zero over $\mathscr{U}_{0}$ and with $H_{p} G>0$ in $p^{-1}(0)_{\mid \mathscr{S}_{0} \backslash \operatorname{neigh}\left(0, \mathbb{R}^{n}\right)}$. After an $\varepsilon$-dependent dilation (see Definition A.5 and the following discussion), [11] applies and using the spaces $H\left(\Lambda_{t G} ; m\right)\left({ }^{4}\right)$, from that work, we have a well-defined operator

$$
P_{\varepsilon}: H\left(\Lambda_{t G} ; \tilde{r}_{\varepsilon}^{2}\right) \longrightarrow H\left(\Lambda_{t G} ; 1\right),
$$

where

$$
\tilde{r}_{\varepsilon}(x, \xi)=\left(r_{\varepsilon}^{2}(x)+\xi^{2}\right)^{\frac{1}{2}} \quad \text { and } \quad r_{\varepsilon}(x)=\left(\frac{\varepsilon+x^{2}}{1+x^{2}}\right)^{\frac{1}{2}}, \quad \forall(x, \xi) \in \mathbb{R}^{2 n} .
$$

See Appendix A for more details. From now on, we denote by $H\left(\Lambda_{t G}\right)$ the space $H\left(\Lambda_{t G} ; 1\right)$.

Let $P_{\varepsilon}^{\text {int }}$ be a suitable self-adjoint reference operator, obtained from $P_{\varepsilon}$ by "filling the sea up to the level $\frac{\varepsilon}{\mathcal{O}(1)}$ ". We have Weyl asymptotics for its spectrum.

$\left({ }^{4}\right)$ Here $m$ is an order function, see (A.21). 


\section{Proposition 2.2. For z satisfying}

$$
-\mathcal{O}(\varepsilon)<\Re z<\frac{\varepsilon}{\mathcal{O}(1)} \quad \text { and } \quad-\frac{\varepsilon}{\mathcal{O}(1)}<\Im z<\mathcal{O}(\varepsilon)
$$

with

$$
\operatorname{dist}\left(z, \sigma\left(P_{\varepsilon}^{\mathrm{int}}\right)\right) \geq \tilde{\delta} \geq h^{N_{0}}
$$

we have

$$
\left(P_{\varepsilon}-z\right)^{-1}=\mathcal{O}\left(\frac{1}{\tilde{\delta}}\right): H\left(\Lambda_{t G}\right) \longrightarrow H\left(\Lambda_{t G}, \tilde{r}_{\varepsilon}^{2}\right) \cap H\left(\Lambda_{t G}\right) .
$$

Inside the set (2.1) there exists a bijection $b: \sigma\left(P_{\varepsilon}^{\mathrm{int}}\right) \longrightarrow \sigma\left(P_{\varepsilon}\right)$ with $b(\mu)-\mu=\mathcal{O}\left(h^{\infty}\right)$.

For $0<\delta \ll 1,0<\varepsilon \leq \varepsilon(\delta), 0<h \leq h(\delta, \varepsilon)$, let $-\mathcal{O}(1)<A<B<\frac{1}{\mathcal{O}(1)}$. By moving the eigenvalues of $P_{\varepsilon}^{\text {int }}$ out of the gaps $\left.A \varepsilon+\right]-\delta \varepsilon, \delta \varepsilon[$ and $B \varepsilon+]-\delta \varepsilon, \delta \varepsilon$ [ we can construct an operator $P_{\varepsilon, \delta}:=P_{\varepsilon, A, B, \delta}: H\left(\Lambda_{t G}, \tilde{r}_{\varepsilon}^{2}\right) \longrightarrow H\left(\Lambda_{t G}\right)$ such that

- the eigenvalues of $P_{\varepsilon, \delta}$ with $-\mathcal{O}(\varepsilon)<\Re z<\frac{\varepsilon}{\mathcal{O}(1)}$ belong to a complex $h^{N_{0}}$-neighborhood of

$$
\begin{aligned}
\left.R_{\varepsilon, A, B, \delta}:=\right]-\mathcal{O}(\varepsilon), \frac{\varepsilon}{\mathcal{O}(1)} & {\left[\backslash(\{A \varepsilon, B \varepsilon\}+]-\frac{\varepsilon \delta}{2}, \frac{\varepsilon \delta}{2}[),\right.} \\
\#\left(\sigma\left(P_{\varepsilon, \delta}\right) \cap(] A \varepsilon, B \varepsilon[+i]-o(\varepsilon), o(\varepsilon)[)\right) & \\
& =\left(\frac{1}{2 \pi h}\right)^{n}(\omega(\varepsilon B)-\omega(\varepsilon A))+\mathcal{O}(\delta \varepsilon) h^{-n} .
\end{aligned}
$$

\subsection{Relative determinants (Sections 11, 12)}

Recall (see e.g. [10]) that under suitable but very general assumptions on the linear operators $\mathcal{A}$ and $\mathcal{B}$,

$$
\begin{aligned}
\left|\operatorname{det} \mathcal{A B}^{-1}\right|=\left|\operatorname{det}\left(1+(\mathcal{A}-\mathcal{B}) \mathcal{B}^{-1}\right)\right| \leq \exp \left\|(\mathcal{A}-\mathcal{B}) \mathcal{B}^{-1}\right\|_{\operatorname{tr}} & \\
& \leq \exp \left(\|\mathcal{A}-\mathcal{B}\|_{\text {tr }}\left\|\mathcal{B}^{-1}\right\|\right) .
\end{aligned}
$$

Here $\|\cdot\|$ denotes the operator norm and $\|\cdot\|_{t r}$ the trace class norm. Let introduce the following sets, (see Figure 2):

$$
\begin{gathered}
R:=]-\mathcal{O}(\varepsilon), \frac{\varepsilon}{\mathcal{O}(1)}[+i]-\frac{\varepsilon}{\mathcal{O}(1)}, \mathcal{O}(\varepsilon)[, \\
R_{\delta}:=\{z \in R ;|\Im z|>\delta \varepsilon\}, \quad \text { and }
\end{gathered}
$$




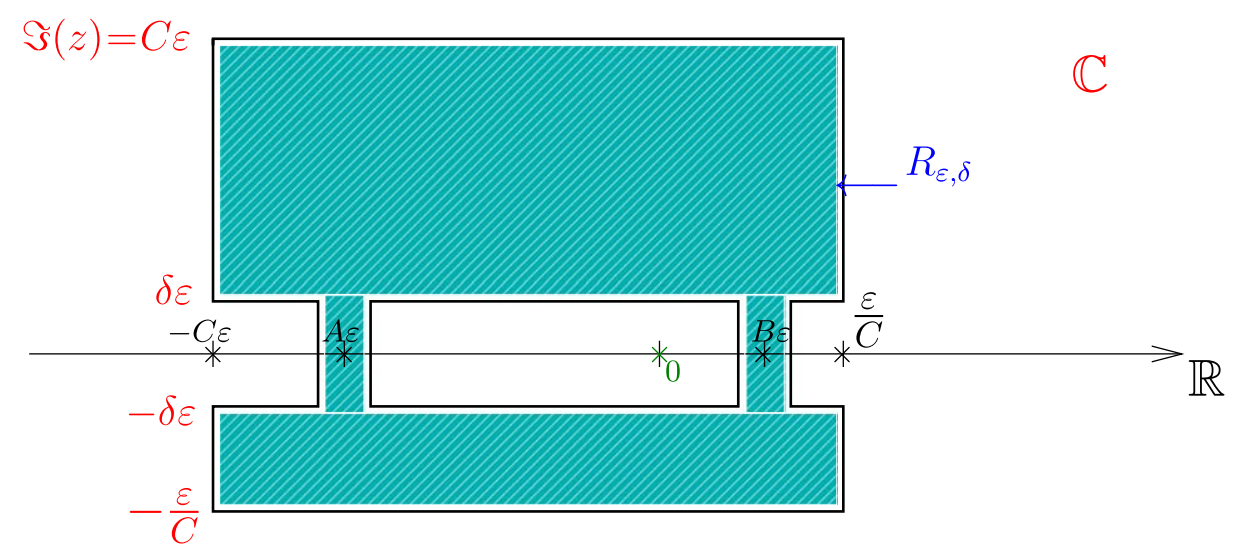

Figure 2. The set $R_{\varepsilon, \delta}$.

$$
\begin{array}{r}
R_{\varepsilon, \delta}:=R_{\delta} \bigcup(A \varepsilon+]-\frac{\delta \varepsilon}{4}, \frac{\delta \varepsilon}{4}[+i[-\delta \varepsilon, \delta \varepsilon]) \\
\bigcup(B \varepsilon+]-\frac{\delta \varepsilon}{4}, \frac{\delta \varepsilon}{4}[+i[-\delta \varepsilon, \delta \varepsilon]) .
\end{array}
$$

We can construct an operator $P_{\varepsilon}^{\text {ext }}$ as a "trace class filling of $P_{\varepsilon}$ over $\mathscr{U}_{0}$ " so that $\left(P_{\varepsilon}^{\text {ext }}-z\right)^{-1}$ is $\mathcal{O}\left(\frac{1}{\varepsilon}\right)$ as a bounded operator from $H\left(\Lambda_{t G}\right)$ to itself, for all $z \in R$. We also have

$$
\begin{gathered}
\left\|P_{\varepsilon}-P_{\varepsilon}^{\text {ext }}\right\|_{\mathrm{tr}}=\mathcal{O}\left(h^{-n}\right), \quad\left\|P-P_{\varepsilon}\right\|_{\mathrm{tr}}=\mathcal{O}\left(\varepsilon^{n+1} h^{-n}\right) \quad \text { and } \\
\left\|P_{\varepsilon}-P_{\varepsilon, \delta}\right\|_{\mathrm{tr}}=\mathcal{O}\left((\varepsilon \delta)^{2} h^{-n}\right) .
\end{gathered}
$$

Define

$$
\begin{aligned}
\mathcal{D}_{P}(z) & =\ln \left|\operatorname{det}(P-z)\left(P_{\varepsilon}^{\text {ext }}-z\right)^{-1}\right| \\
\mathcal{D}_{P_{\varepsilon}}(z) & =\ln \left|\operatorname{det}\left(P_{\varepsilon}-z\right)\left(P_{\varepsilon}^{\text {ext }}-z\right)^{-1}\right| \\
\mathcal{D}_{P_{\varepsilon, \delta}}(z) & =\ln \left|\operatorname{det}\left(P_{\varepsilon, \delta}-z\right)\left(P_{\varepsilon}^{\text {ext }}-z\right)^{-1}\right| .
\end{aligned}
$$

The zeros of $\operatorname{det}\left((P-z)\left(P_{\varepsilon}^{\mathrm{ext}}-z\right)^{-1}\right)$ coincide with the resonances of $P$. We have

$$
\mathcal{D}_{P}-\mathcal{D}_{P_{\varepsilon}} \begin{cases}\leq \mathcal{O}_{\delta}(1) \varepsilon^{n} h^{-n} & \text { in } R_{\delta}, \\ \geq-\mathcal{O}_{\delta}(1) \varepsilon^{n} h^{-n} & \text { in } R_{\delta} \cap\left\{z \in \mathbb{C} ; \Re z \leq-\frac{\varepsilon}{\mathcal{O}(1)}\right\} .\end{cases}
$$

Similar estimates hold for $\mathcal{D}_{P}-\mathcal{D}_{P_{\varepsilon, \delta}}$ with $\varepsilon^{n} h^{-n}$ replaced by $\varepsilon \delta h^{-n}$ and $R_{\delta}$ by $R_{\varepsilon, \delta}$.

Standard arguments, including Jensen's formula, lead to 


\section{Proposition 2.3.}

(A) The number of resonances in $R_{\delta}$ is $\leq \mathcal{O}_{\delta}(1) \varepsilon^{n} h^{-n}$ with the usual convention that $0<\varepsilon \leq \varepsilon(\delta), 0<h \leq h(\delta, \varepsilon)$.

(B) There are plenty of $z \in R_{\delta}$ for which

$$
\mathcal{D}_{P}(z)-\mathcal{D}_{P_{\varepsilon}}(z) \geq-\mathcal{O}_{\delta}(1) \varepsilon^{n} h^{-n} .
$$

The point $(A)$ of the above proposition gives part $(A)$ in Theorem 1.1.

\section{Proposition 2.4.}

(A) The number of resonances of $P$ in $R_{\varepsilon, \delta}$ is $\leq \mathcal{O}(\varepsilon \delta) h^{-n}$.

(B) There are plenty of $z \in R_{\varepsilon, \delta}$ for which

$$
\mathcal{D}_{P}(z)-\mathcal{D}_{P_{\varepsilon, \delta}}(z) \geq-\mathcal{O}(\varepsilon \delta) h^{-n} .
$$

Consider the holomorphic function $f(z)=\operatorname{det}\left((P-z)\left(P_{\varepsilon}^{\text {ext }}-z\right)^{-1}\right)$ on $R$. Then

$$
|f(z)| \leq \exp \left(h^{-n}(\phi(z)+\mathcal{O}(\varepsilon \delta))\right) \quad \text { in } R_{\varepsilon, \delta},
$$

where $\phi(z)=h^{n} \mathcal{D}_{P_{\varepsilon, \delta}}$. By $(B)$ in Proposition 2.4 we have

$$
|f(z)| \geq \exp \left(h^{-n}(\phi(z)-\mathcal{O}(\varepsilon \delta))\right) \text { at plenty of points in } R_{\varepsilon, \delta} .
$$

We can then apply Theorem 1.1 in [16] (or [17, Theorem 12.1.1]) with $h$ there replaced by $h^{n}$, to finish the proof.

\section{Escape functions}

From the property (1.11), we get after an orthogonal change of $x$-variables and a subsequent dilation in the variable $x_{n}$,

$$
p(x, \xi)=\frac{\varkappa}{2}\left(\xi_{n}^{2}-x_{n}^{2}\right)+\frac{1}{2} q\left(x^{\prime}, \xi^{\prime}\right)+\mathcal{O}\left(x^{3}\right),
$$

where $\varkappa$ is a positive constant. Here we write $x=\left(x^{\prime}, x_{n}\right) \in \mathbb{R}^{n}, x^{\prime}=\left(x_{1}, \ldots, x_{n-1}\right) \in$ $\mathbb{R}^{n-1}$ and similarly for the dual variable $\xi$. The quadratic form $q$ is positive definite. For simplicity, we may assume that $\varkappa=1$ :

$$
p(x, \xi)=\frac{1}{2}\left(\xi_{n}^{2}-x_{n}^{2}\right)+\frac{1}{2} q\left(x^{\prime}, \xi^{\prime}\right)+\mathcal{O}\left(|x|^{3}\right),
$$

for $x \in \operatorname{neigh}\left(0, \mathbb{R}^{n}\right), \xi \in \mathbb{R}^{n}$. Let

$$
p_{0}=\frac{1}{2}\left(\xi_{n}^{2}-x_{n}^{2}\right) \quad \text { and } \quad G_{0}=x_{n} \xi_{n} .
$$


Then

$$
H_{p_{0}}=\left(\partial_{\xi_{n}} p_{0}\right) \partial_{x_{n}}-\left(\partial_{x_{n}} p_{0}\right) \partial_{\xi_{n}}
$$

and

$$
H_{p_{0}} G_{0}=x_{n}^{2}+\xi_{n}^{2}
$$

Let $\Psi \in C^{\infty}(\mathbb{R} ;[0,1])$ have its support $\left.\left.=\right]-\infty, 1\right]$ and be equal to one on ]$\left.-\infty, \frac{1}{2}\right]$. For $\lambda \geq 1$ large enough (to be fixed below), we put

$$
G(x, \xi)=G^{\varepsilon}(x, \xi)=\left(1-\Psi\left(\frac{\lambda x_{n}}{\sqrt{\varepsilon+x^{\prime 2}+\xi^{2}}}\right)\right) G_{0}(x, \xi) .
$$

Here $\varepsilon>0$ is a small parameter with respect to which our estimates will be uniform. We will consider $G$ for $(x, \xi) \in \operatorname{neigh}\left(0, \mathbb{R}^{2 n}\right), \xi \in \mathbb{R}^{n}$ and notice first that $G=0$ for $x_{n}<0$, so we may restrict the attention to the region $x_{n} \geq 0$.

The prefactor $1-\Psi\left(\lambda x_{n}\left(\varepsilon+x^{\prime 2}+\xi^{2}\right)^{-\frac{1}{2}}\right)$ in (3.5) is smooth and positively homogeneous of degree 0 in the variables $(\sqrt{\varepsilon}, \rho)=(\sqrt{\varepsilon}, x, \xi)$. It follows that in $\operatorname{neigh}\left(0, \mathbb{R}^{2 n}\right)$

$$
\partial_{\rho}^{\alpha} G=\mathcal{O}(1)\left(\varepsilon+\rho^{2}\right)^{1-\frac{|\alpha|}{2}}, \quad \alpha \in \mathbb{N}^{2 n} .
$$

For $|\alpha|=0,1$, we have

$$
G=\mathcal{O}(1)|\rho|^{2}, \quad \partial_{\rho} G=\mathcal{O}(|\rho|)
$$

Notice that the support of $G$ is contained in the region where

$$
x_{n}^{2} \geq \frac{1}{4 \lambda^{2}}\left(\varepsilon+\left(x^{\prime}, \xi^{\prime}\right)^{2}+\xi_{n}^{2}\right) .
$$

In the cutoff region, where the prefactor in $(3.5)$ is $\neq 1$, we have

$$
x_{n}^{2} \leq \frac{1}{\lambda^{2}}\left(\varepsilon+\left(x^{\prime}, \xi^{\prime}\right)^{2}+\xi_{n}^{2}\right) .
$$

Since the quadratic form $q$ is positive definite,

$$
q\left(x^{\prime}, \xi^{\prime}\right) \geq \frac{1}{C}\left(x^{\prime}, \xi^{\prime}\right)^{2},
$$

we get from (3.1), (3.9), that in the cutoff region,

$$
p \geq \frac{1}{2}\left(\xi_{n}^{2}+\frac{1}{C}\left(x^{\prime}, \xi^{\prime}\right)^{2}\right)-\frac{1}{2 \lambda^{2}}\left(\varepsilon+\left(x^{\prime}, \xi^{\prime}\right)^{2}+\xi_{n}^{2}\right)-\mathcal{O}\left(|x|^{3}\right) .
$$


In view of (3.9) we can add the non-positive term

$$
\frac{1}{2} x_{n}^{2}-\frac{1}{2 \lambda^{2}}\left(\varepsilon+\left(x^{\prime}, \xi^{\prime}\right)^{2}+\xi_{n}^{2}\right)
$$

to the right hand side and get in the cutoff region,

$$
p \geq \frac{1}{2}\left(x_{n}^{2}+\xi_{n}^{2}+\frac{1}{C}\left(x^{\prime}, \xi^{\prime}\right)^{2}\right)-\frac{1}{\lambda^{2}}\left(\varepsilon+\left(x^{\prime}, \xi^{\prime}\right)^{2}+\xi_{n}^{2}\right)-\mathcal{O}\left(|x|^{3}\right) .
$$

Assume for simplicity that $C \geq 1$ and fix $\lambda \geq 1$ such that

$$
\frac{1}{\lambda^{2}}<\frac{1}{4 C} \text {. }
$$

Absorbing the term $-\mathcal{O}\left(|x|^{3}\right)$ by restricting the attention to a small neighborhood of $x=0$, we get in the cutoff region (that is the one where the prefactor in (3.5) is $\neq 1)$,

$$
p \geq-\frac{\varepsilon}{4 C}+\frac{1}{4} x_{n}^{2}+\frac{1}{4} \xi_{n}^{2}+\frac{1}{4 C}\left(x^{\prime}, \xi^{\prime}\right)^{2},
$$

hence with a new constant $C \geq 1$ :

$$
p(\rho) \geq-\frac{\varepsilon}{C}+\frac{\rho^{2}}{C} .
$$

Outside the cutoff region, we have $G=G_{0}$ and hence

$$
H_{p} G=H_{p} G_{0}=H_{p_{0}} G_{0}+\mathcal{O}\left(|x|^{2}\right) \partial_{\xi_{n}} G_{0},
$$

So

$$
H_{p} G=x_{n}^{2}+\xi_{n}^{2}+\mathcal{O}\left(|x|^{2}\right) x_{n} .
$$

Here, we also have $\lambda x_{n} \geq\left(\varepsilon+\left(x^{\prime}, \xi^{\prime}\right)^{2}+\xi_{n}^{2}\right)^{\frac{1}{2}}$, where $\lambda \geq 1$ is now fixed and hence we have outside the cutoff region and inside a small neighborhood of $(0,0)$ :

$$
H_{p} G \asymp \varepsilon+|\rho|^{2} \text {. }
$$

Notice that if

$$
p(\rho)<-\frac{\varepsilon}{C}+\frac{\rho^{2}}{C}
$$

then by (3.14) we are outside the cutoff region and (3.16) holds.

Proposition 3.1. Let $\lambda>0$ be sufficiently large in the definition (3.5) of $G^{\varepsilon}$ and let $(\rho, \varepsilon)$ vary in neigh $\left.\left.\left(0, \mathbb{R}^{2 n}\right) \times\right] 0, \varepsilon_{0}\right]$ for $\varepsilon_{0}>0$ sufficiently small. Then there exists a constant $C \geq 1$ such that if (3.17) holds, then (3.16) holds uniformly for $\rho \in \operatorname{neigh}\left(0, \mathbb{R}^{2 n}\right)$.

If $p(\rho)<-\frac{\varepsilon}{\widetilde{C}}+\frac{\rho^{2}}{C}$ for some fixed $\widetilde{C} \geq C$, we reach the same conclusion if we replace $G^{\varepsilon}$ by $G^{\tilde{\varepsilon}}$ where $\tilde{\varepsilon}=\frac{C}{\widetilde{C}} \varepsilon$. 


\section{Adding a bump at the saddle point}

Let $\chi(x, \xi)>0$ be an analytic function on $\mathbb{R}^{2 n}$ with a holomorphic extension to the domain

$$
|\Im(x, \xi)|<\frac{1}{C}\langle\Re(x, \xi)\rangle
$$

satisfying

$$
\chi(x, \xi)=\mathcal{O}(1) \exp \left(-\frac{1}{C}(\Re(x, \xi))^{2}\right),
$$

where the extension is denoted by the same symbol. We use the standard notation $\langle\rho\rangle=\left(1+\rho^{2}\right)^{\frac{1}{2}}$ for real vectors $\rho$.

For $0<\varepsilon \ll 1$, we put

$$
\chi_{\varepsilon}(x, \xi)=\varepsilon \chi\left(\frac{x}{\sqrt{\varepsilon}}, \frac{\xi}{\sqrt{\varepsilon}}\right) .
$$

Assume for simplicity that

$$
\chi \leq 1 \quad \text { on the real domain. }
$$

Then

$$
\chi_{\varepsilon} \leq \varepsilon \quad \text { on the real domain. }
$$

For every $r_{0}>0$, there exists $a_{0}=a_{0}\left(r_{0}, \chi\right)>0$ such that

$$
\chi \geq a_{0} \quad \text { on } B_{\mathbb{R}^{2 n}}\left(0, r_{0}\right) .
$$

Since $\chi$ is positive, we get

$$
\chi(x, \xi) \geq a\left(1-\frac{x^{2}+\xi^{2}}{r_{0}^{2}}\right) \quad \text { on } \mathbb{R}^{2 n} \text {, for } 0 \leq a \leq a_{0} .
$$

Hence for the same values of $a$

$$
\chi_{\varepsilon}(x, \xi) \geq a\left(\varepsilon-\frac{x^{2}+\xi^{2}}{r_{0}^{2}}\right),
$$

on the real domain.

Let

$$
p_{\varepsilon}=p+\chi_{\varepsilon} .
$$

Then, by (4.5),

$$
p_{\varepsilon} \leq p+\varepsilon .
$$


Assume that

$$
p_{\varepsilon}(\rho) \leq b \varepsilon+c \rho^{2},
$$

where $b, c>0$ are constants to be chosen below. Using (4.6), (4.7), we have

$$
p(\rho)=p_{\varepsilon}(\rho)-\chi_{\varepsilon}(\rho) \leq b \varepsilon+c \rho^{2}-a \varepsilon+\frac{a}{r_{0}^{2}} \rho^{2},
$$

i.e.

$$
p(\rho) \leq(b-a) \varepsilon+\left(c+\frac{a}{r_{0}^{2}}\right) \rho^{2} .
$$

Let $C \geq 1$ be the constant in Proposition 3.1. If for some constant $\widetilde{C} \geq C$,

$$
b-a \leq-\frac{1}{\widetilde{C}}, \quad c+\frac{a}{r_{0}^{2}} \leq \frac{1}{C}
$$

we get

$$
p(\rho) \leq-\frac{\varepsilon}{\widetilde{C}}+\frac{\rho^{2}}{C}
$$

and hence (3.16) holds for $G=G^{\tilde{\varepsilon}}$ with $\tilde{\varepsilon}=\frac{C}{\widetilde{C}} \varepsilon$ in (3.5) (as we saw in Proposition 3.1)

$$
H_{p} G \asymp \varepsilon+\rho^{2} .
$$

For a given $r_{0}>0$, we know that (4.7) holds for $0<a \leq a_{0}$ for some $a_{0}>0$. Choose $a$ so that $\frac{a}{r_{0}^{2}} \leq \frac{1}{2 C}$ and put $c=\frac{1}{2 C}$. Then the second inequality in (4.12) is valid. Choose $b=\frac{a}{2}$ and $\widetilde{C} \geq C$ large enough. Then the first estimate in (4.12) also holds. With this choice of $a, b, c$, we know that (4.10) implies (4.13) and hence also (3.16) $(\Longleftrightarrow(4.14))$, for $G=G^{\frac{C \varepsilon}{\tilde{C}}}$.

After the dilation $\chi(\rho) \mapsto \chi(\alpha \rho), \alpha \in] 0,1],(4.7)$ remains valid. Hence we still have that $(4.10) \Longrightarrow(4.14)(\Longleftrightarrow(3.16))$ (with the same fixed dilation in $\varepsilon$ ).

We next study

$$
\begin{aligned}
H_{p_{\varepsilon}} G & =H_{p} G+\left\{\varepsilon \chi\left(\frac{x}{\sqrt{\varepsilon}}, \frac{\xi}{\sqrt{\varepsilon}}\right), G\right\} \\
& =H_{p} G+\mathcal{O}(1)\left|(\nabla \chi)\left(\frac{x}{\sqrt{\varepsilon}}, \frac{\xi}{\sqrt{\varepsilon}}\right)\right| \sqrt{\varepsilon}|(x, \xi)|,
\end{aligned}
$$

where we used (3.7) in the last step. Here $\{f, g\}=H_{f} g$ denotes the Poisson bracket of two $C^{1}$ functions $f, g$. Thus

$$
H_{p_{\varepsilon}} G=H_{p} G+\mathcal{O}(1)\|\nabla \chi\|_{L^{\infty}}\left(\varepsilon+\rho^{2}\right) .
$$


Replacing $\chi$ with $\chi(\alpha \rho)$, gives

$$
H_{p_{\varepsilon}} G=H_{p} G+\mathcal{O}(1) \alpha\left(\varepsilon+\rho^{2}\right) .
$$

Choose $a, b, c$ as in the preceding discussion and $\alpha>0$ small enough in

$$
p_{\varepsilon}(\rho)=p(\rho)+\varepsilon \chi\left(\varepsilon^{-\frac{1}{2}} \alpha \rho\right) .
$$

Then in the region (4.10) we have

$$
H_{p_{\varepsilon}} G \asymp \varepsilon+\rho^{2}, \quad G=G^{\tilde{\varepsilon}}, \quad \tilde{\varepsilon}=\frac{C}{\widetilde{C}} \varepsilon .
$$

Proposition 4.1. Define $p_{\varepsilon}=p+\chi_{\varepsilon}$ as in (4.2), (4.1), (4.3), (4.4). Assume also that $\|\nabla \chi\| \leq \alpha_{0}$ for some sufficiently small $\alpha_{0}>0$. Let $G^{\varepsilon}$ be as in Proposition (3.1) and let $(\rho, \varepsilon)$ vary in neigh $\left.\left.\left(0, \mathbb{R}^{2 n}\right) \times\right] 0, \varepsilon_{0}\right]$ for $\varepsilon_{0}>0$ sufficiently small. Then there exist constants $b, c>0$ and $\widetilde{C} \geq C>0$ such that if (4.10) holds, then we have (4.13), and (4.18) holds uniformly for $\rho \in \operatorname{neigh}\left(0, \mathbb{R}^{2 n}\right)$.

\section{Deformed phase space}

We continue to work with the function $G$ of Section 3 , where $\lambda \geq 1$ now is fixed and $\varepsilon$ is a small parameter. $G$ vanishes near $\left\{(x, \xi) \in\right.$ neigh $\left.\left(0, \mathbb{R}^{2 n}\right) ; x_{n} \leq 0\right\}$ and we restrict the attention to the set $x_{n} \geq 0$. We saw in Proposition 3.1 that there is a constant $C \geq 1$ such that

$$
(3.17) \Longrightarrow(3.16)
$$

uniformly. Also if $\widetilde{C} \geq C$, the estimate

$$
p(\rho) \leq-\frac{\varepsilon}{\widetilde{C}}+\frac{\rho^{2}}{C}
$$

implies (3.16) uniformly, where $G=G^{\frac{C \varepsilon}{C}}$.

For $0 \leq t \ll 1$, we introduce the IR-manifold (see Appendix A)

$$
\Lambda_{t G}=\left\{\rho+i t H_{G}(\rho) ; \rho \in \operatorname{neigh}\left(0, \mathbb{R}^{2 n}\right)\right\} .
$$

By Taylor expansion, we have

$$
\begin{gathered}
\Im p\left(\rho+i t H_{G}(\rho)\right)=-t H_{p} G(\rho)+\mathcal{O}\left(t^{3}|\rho|^{3}\right), \\
\Re p\left(\rho+i t H_{G}(\rho)\right)=p(\rho)+\mathcal{O}\left(t^{2} \rho^{2}\right) .
\end{gathered}
$$

Here we also use that $\left|H_{G}(\rho)\right|=\left|\partial_{\rho} G\right|=\mathcal{O}(|\rho|)$ by (3.7). 
Proposition 5.1. Let $\widetilde{C} \geq C>0$ be as above and let $G=G^{\frac{C \varepsilon}{\widetilde{C}}}$. Then if $t>0$ is small enough, we have that

$$
\Re p\left(\rho+i t H_{G}(\rho)\right) \leq-\frac{\varepsilon}{\widetilde{C}}+\frac{\rho^{2}}{2 C}
$$

implies that

$$
\Im p\left(\rho+i t H_{G}(\rho)\right) \asymp-t\left(\rho^{2}+\varepsilon\right) .
$$

We recall that we work in neigh $\left(0, \mathbb{R}^{2 n}\right) \cap\left\{x_{n} \geq 0\right\}$.

Proof. From (5.5) we get by means of (5.4),

$$
p(\rho) \leq-\frac{\varepsilon}{\widetilde{C}}+\left(\frac{1}{2 C}+\mathcal{O}\left(t^{2}\right)\right) \rho^{2}
$$

and hence (5.1):

$$
p(\rho) \leq-\frac{\varepsilon}{\widetilde{C}}+\frac{\rho^{2}}{C},
$$

if $t$ is small enough. Then by (3.16), (5.3) we get

$$
\begin{aligned}
\Im p\left(\rho+i t H_{G}(\rho)\right) & =-t H_{p} G(\rho)+\mathcal{O}\left(t^{3}|\rho|^{3}\right) \\
& =-t\left(H_{p} G+\mathcal{O}\left(t^{2}\right)|\rho|^{3}\right) \\
& \asymp-t\left(\varepsilon+\rho^{2}\right) . \quad \square
\end{aligned}
$$

We next turn to $p_{\varepsilon}$ and recall Proposition 4.1. From (4.2) and the Cauchy inequalities, we get after slightly increasing the constant $C=C_{\chi}>0$ there:

$$
\partial_{\rho}^{\alpha} \chi(\rho)=\mathcal{O}(1) \exp \left(-\frac{1}{C}(\Re \rho)^{2}\right), \quad|\Im \rho|<\frac{1}{C}\langle\Re \rho\rangle .
$$

For $\chi_{\varepsilon}($ cf. $(4.3))$ we get

$$
\partial_{\rho}^{\alpha} \chi_{\varepsilon}(\rho)=\mathcal{O}(1) \varepsilon^{1-\frac{|\alpha|}{2}} \exp \left(-\frac{1}{C \varepsilon}(\Re \rho)^{2}\right),
$$

when

$$
|\Im \rho| \leq \frac{1}{C}(\sqrt{\varepsilon}+|\Re \rho|) .
$$

In particular, we have

$$
\partial_{\rho}^{\alpha} \chi_{\varepsilon}(\rho)=\mathcal{O}(1) \exp \left(-\frac{1}{C \varepsilon}(\Re \rho)^{2}\right), \quad \text { when }|\alpha| \leq 2 .
$$


Since $H_{G}=\mathcal{O}(\rho), \Lambda_{t G}$ is included in the region (5.11) when $0 \leq t \ll 1$ and by Taylor expansion we get

$$
\begin{aligned}
-\Im p_{\varepsilon}\left(\rho+i t H_{G}(\rho)\right) & =t H_{p_{\varepsilon}} G+\mathcal{O}\left(1+\varepsilon^{-\frac{1}{2}} e^{-\frac{\rho^{2}}{C \varepsilon}}\right) t^{3}|\rho|^{3} \\
& =t H_{p_{\varepsilon}} G+\mathcal{O}(1) t^{3}|\rho|^{2} .
\end{aligned}
$$

Similarly,

$$
\Re p_{\varepsilon}\left(\rho+i t H_{G}(\rho)\right)=p_{\varepsilon}(\rho)+\mathcal{O}(1) t^{2} \rho^{2} .
$$

This is analogous to (5.3), (5.4) and we get

Proposition 5.2. Let b, $c, C, \widetilde{C}>0$ be as in Proposition 4.1, choose $G$ as in (4.18). Then for $0 \leq t \ll 1$, if

$$
\Re p_{\varepsilon}\left(\rho+i t H_{G}(\rho)\right) \leq b \varepsilon+\frac{c}{2} \rho^{2},
$$

we have (4.10):

$$
p_{\varepsilon}(\rho) \leq b \varepsilon+c \rho^{2},
$$

and we conclude as in Proposition 4.1 that (4.13), (4.18) hold:

$$
\begin{gathered}
p(\rho) \leq-\frac{\varepsilon}{\widetilde{C}}+\frac{\rho^{2}}{C}, \\
H_{p_{\varepsilon}} G \asymp \varepsilon+\rho^{2} .
\end{gathered}
$$

Hence by (5.13),

$$
-\Im p_{\varepsilon}\left(\rho+i t H_{G}(\rho)\right) \asymp t\left(\varepsilon+\rho^{2}\right),
$$

when $0<t \ll 1$. Recall here that $\rho \in \operatorname{neigh}\left(0, \mathbb{R}^{2 n}\right)$ with $x_{n}(\rho) \geq 0$.

Define $r(x), R(x)$ as in (A.8) below:

$$
r(x)=1, \quad R(x)=\langle x\rangle,
$$

and put

$$
\tilde{r}(x, \xi)=\left(r(x)^{2}+\xi^{2}\right)^{\frac{1}{2}} .
$$

Define the class $\dot{S}\left(\mathbb{R}^{2 n} ; R \tilde{r}\right.$ ) as in Definition A.2 (b) (see [11, Chapter 1, Définition 1.4]).( $\left(^{5}\right)$ From the appendix in $[9]$ we see that if $\Psi_{0} \in C_{0}^{\infty}\left(\mathbb{R}^{2 n} ;[0,1]\right)$ is equal to one

$\left(^{5}\right)$ For our special choice of $r, R, \dot{S}\left(\mathbb{R}^{2 n} ; R \tilde{r}\right)=S\left(\mathbb{R}^{2 n} ; R \tilde{r}\right)$, but we prefer $\dot{S}\left(\mathbb{R}^{2 n} ; R \tilde{r}\right)$ as in the general theory, allowing for more general scales near infinity. 
near $(0,0)$, then there exists $\widetilde{G}=\Psi_{0} G+F$, where $F$ is independent of $\varepsilon$,

$$
\begin{gathered}
F \in \dot{S}(\tilde{r} R), \quad F=0 \quad \text { when }|\xi| \gg r(x), \\
(0,0) \notin \operatorname{supp} F, \\
H_{p} \widetilde{G}>0 \quad \text { on } p^{-1}(0) \cap\left(\overline{\mathscr{S}}_{0} \times \mathbb{R}^{n} \backslash\{(0,0)\}\right),
\end{gathered}
$$

and uniformly $\geq \frac{1}{\mathcal{O}(1)}$ outside any fixed neighborhood of $(0,0)$ in $\left(\overline{\mathscr{S}}_{0} \times \mathbb{R}^{n}\right) \backslash\{(0,0)\}$. We can also arrange so that $\pi_{x}(\operatorname{supp} F)$ is contained in an arbitrarily small neighborhood of $\overline{\mathscr{S}}_{0}\left(\pi_{x}\right.$ is introduced in Footnote 3 in Section 1$)$.

By Taylor expansion we see that

- In a small fixed neighborhood of $(0,0)$ we have $\widetilde{G}=G$ and the Propositions $5.1,5.2$ hold with $G$ replaced by $\widetilde{G}$.

- Away from any fixed neighborhood of $(0,0)$ and for any fixed $\left.t \in] 0, t_{0}\right]$ with $t_{0}>0$ small enough, we have

$$
\left\{\begin{array}{l}
p(\rho)=0, \\
\pi_{x}(\rho) \in \mathscr{S}_{0}
\end{array} \Longrightarrow-\Im p\left(\left(\rho+i t H_{\widetilde{G}}(\rho)\right) \asymp t,\right.\right.
$$

uniformly in $\varepsilon$. Also, since $\Re p\left(\rho+i t H_{\widetilde{G}}(\rho)\right)=p(\rho)+\mathcal{O}\left(t^{2}\right)$, we conclude that away from any small fixed neighborhood of $(0,0)$, we have

$$
\frac{t}{C} \tilde{r}^{2} \leq\left|p\left(\rho+i t H_{\widetilde{G}}(\rho)\right)\right| \leq C \tilde{r}^{2} .
$$

and $p\left(\rho+\left.i t H_{\widetilde{G}}(\rho)\right|_{\overline{\mathscr{S}}_{0} \times \mathbb{R}^{n}}\right.$ is an elliptic symbol of class $S\left(\mathbb{R}^{2 n} ; \tilde{r}^{2}\right)$ away from any fixed neighborhood of $(0,0)$.

\section{Preparations for the study of $P_{\varepsilon}$}

Let

$$
P=-h^{2} \Delta+V(x)
$$

so that $P$ is the $h$-Weyl quantization of the symbol $p(x, \xi)=\xi^{2}+V(x)$. Recall the definition of the symbol $\chi_{\varepsilon}(x, \xi)$ in $(4.3)$. By $\chi_{\varepsilon}$ we will also denote a suitable $h$-quantization (very close to the Weyl-one). Let

$$
P_{\varepsilon}=P+\chi_{\varepsilon}
$$

be the corresponding quantization of

$$
p_{\varepsilon}=p+\chi_{\varepsilon}=\xi^{2}+V(x)+\chi_{\varepsilon}(x, \xi) .
$$


Assume for simplicity that

$$
\partial_{\xi} \chi(x, 0)=0
$$

a condition which is fulfilled in the main case that we have in mind:

$$
\chi(x, \xi)=\exp \left(-\frac{1}{C}(x, \xi)^{2}\right) .
$$

Recall that in Section 4, we have replaced $\chi(\rho)$ by $\chi(\alpha \rho)$ for some sufficiently small fixed $\alpha>0$, in order to have Proposition 4.1 available. With $\alpha$ small enough, we get from (6.4) that

$$
\inf _{\xi \in \mathbb{R}^{n}}\left(\frac{\xi^{2}}{2}+\chi_{\varepsilon}(x, \xi)\right)=\chi_{\varepsilon}(x, 0) .
$$

This follows from the fact that

$$
\partial_{\rho}^{2} \chi_{\varepsilon}=\mathcal{O}\left(\alpha^{2}\right), \quad \partial_{\rho} \chi_{\varepsilon}=\mathcal{O}(\sqrt{\varepsilon} \alpha), \quad \chi_{\varepsilon}=\mathcal{O}(\varepsilon)
$$

(We could here replace $\frac{\xi^{2}}{2}$ by $\theta \xi^{2}$ for any $0<\theta<1$ if $\alpha=\alpha(\theta)$ is small enough.)

As a natural potential associated to $P_{\varepsilon}$, we put

$$
V_{\varepsilon}(x)=V(x)+\chi_{\varepsilon}(x, 0)=\inf _{\xi \in \mathbb{R}^{n}}\left(\frac{\xi^{2}}{2}+V(x)+\chi_{\varepsilon}(x, \xi)\right) .
$$

Using (6.7), we see that $V_{\varepsilon}$ is a small perturbation of $V$ in $C^{2}$ and has a critical point $x_{c}(\varepsilon)=\mathcal{O}(\sqrt{\varepsilon})$ which is uniformly non-degenerate of signature $(n-1,1)$. Also,

$$
V_{\varepsilon}\left(x_{c}(\varepsilon)\right) \asymp \varepsilon \text {. }
$$

Assume for simplicity that

$$
\partial \chi(0)=0
$$

Then $x_{c}(\varepsilon)=0$ and

$$
V_{\varepsilon}\left(x_{c}(\varepsilon)\right)=\varepsilon \chi(0)=: E_{\varepsilon} .
$$

In analogy with (1.8), we have

$$
V_{\varepsilon}^{-1}(]-\infty, E_{\varepsilon}[)=\mathscr{U}_{\varepsilon} \sqcup \mathscr{S}_{\varepsilon},
$$

where $\mathscr{U}_{\varepsilon}, \mathscr{S}_{\varepsilon}$ are open, connected and mutually disjoint. Let $\mathscr{U}_{\varepsilon}$ be the bounded component and $\mathscr{S}_{\varepsilon}$ the unbounded one. Again,

$$
\overline{\mathscr{U}}_{\varepsilon} \cap \overline{\mathscr{S}}_{\varepsilon}=\{0\} .
$$


In an $\mathcal{O}(\sqrt{\varepsilon})$-neighborhood of 0 , we write $x=\sqrt{\varepsilon} \tilde{x}$ and

$$
\begin{aligned}
V_{\varepsilon}(x)-E_{\varepsilon} & =V(\sqrt{\varepsilon} \tilde{x})+\varepsilon(\chi(\alpha \tilde{x}, 0)-\chi(0,0)) \\
& =\varepsilon\left(\frac{V(\sqrt{\varepsilon} \tilde{x})}{\varepsilon}+\chi(\alpha \tilde{x}, 0)-\chi(0,0)\right) .
\end{aligned}
$$

Thus, with $V_{0}(\tilde{x})=\left\langle\frac{1}{2} V^{\prime \prime}(0) \tilde{x}, \tilde{x}\right\rangle$,

$$
V_{\varepsilon}(x)-E_{\varepsilon}=\varepsilon\left(V_{0}(\tilde{x})+\mathcal{O}\left(\sqrt{\varepsilon}+\alpha^{2}\right) \tilde{x}^{2}\right) \quad \text { in } C^{\infty} .
$$

Here, we may assume (cf. (3.1)) that

$$
V_{0}(\tilde{x})=q\left(\tilde{x}^{\prime}\right)-\tilde{x}_{n}^{2}
$$

where $q\left(\tilde{x}^{\prime}\right)$ is a positive definite quadratic form and (6.15) gives

$$
V_{\varepsilon}(x)-E_{\varepsilon}=\varepsilon\left(q\left(\tilde{x}^{\prime}\right)-\tilde{x}_{n}^{2}+\mathcal{O}\left(\sqrt{\varepsilon}+\alpha^{2}\right) \tilde{x}^{2}\right) .
$$

For $-\mathcal{O}(\varepsilon) \leq E \leq E_{\varepsilon}$ we have

$$
V_{\varepsilon}^{-1}(]-\infty, E[)=\mathscr{U}_{\varepsilon}(E) \cup \mathscr{S}_{\varepsilon}(E),
$$

where $\mathscr{U}_{\varepsilon}(E) \subset \mathscr{U}_{\varepsilon}=\mathscr{U}_{\varepsilon}\left(E_{\varepsilon}\right), \mathscr{S}_{\varepsilon}(E) \subset \mathscr{S}_{\varepsilon}=\mathscr{S}_{\varepsilon}\left(E_{\varepsilon}\right)$.

Orient the $\tilde{x}_{n}$-axis so that $\tilde{x}_{n}<0$ in $\mathscr{U}_{\varepsilon}$ and $\tilde{x}_{n}>0$ in $\mathscr{S}_{\varepsilon}$. Write $E=E_{\varepsilon}-\varepsilon F$, $0 \leq F \leq \mathcal{O}(1)$. Then on $\partial \mathscr{U}_{\varepsilon}(E) \cup \partial \mathscr{S}_{\varepsilon}(E)$, we have by $(6.17)$,

$$
\begin{gathered}
-F=-\left(1+\mathcal{O}\left(\sqrt{\varepsilon}+\alpha^{2}\right)\right) \tilde{x}_{n}^{2}+\left(1+\mathcal{O}\left(\sqrt{\varepsilon}+\alpha^{2}\right)\right) q\left(\tilde{x}^{\prime}\right), \\
\tilde{x}_{n}= \pm\left(1+\mathcal{O}\left(\sqrt{\varepsilon}+\alpha^{2}\right)\right)\left(F+\left(1+\mathcal{O}\left(\sqrt{\varepsilon}+\alpha^{2}\right)\right) q\left(\tilde{x}^{\prime}\right)\right)^{\frac{1}{2}}
\end{gathered}
$$

Here the plus and minus sign give the local parametrizations of $\partial \mathscr{S}_{\varepsilon}(E)$ and $\partial \mathscr{U}_{\varepsilon}(E)$ respectively. In the original coordinates $x=\sqrt{\varepsilon} \tilde{x}$, this gives

$$
x_{n}= \pm\left(1+\mathcal{O}\left(\sqrt{\varepsilon}+\alpha^{2}\right)\right)\left(\varepsilon F+\left(1+\mathcal{O}\left(\sqrt{\varepsilon}+\alpha^{2}\right)\right) q\left(x^{\prime}\right)\right)^{\frac{1}{2}}
$$

which is a detailed description of $\partial \mathscr{U}_{\varepsilon}(E)$ and $\partial \mathscr{S}_{\varepsilon}(E)$ in any $\mathcal{O}(\sqrt{\varepsilon})$-neighborhood of 0 . The two sets come closest to each other when $\left|x^{\prime}\right| \ll \sqrt{\varepsilon}$ and the distance is

$$
2\left(1+\mathcal{O}\left(\sqrt{\varepsilon}+\alpha^{2}\right)\right)(\varepsilon F)^{\frac{1}{2}}
$$

Moreover, for $E=E_{\varepsilon}-\varepsilon F$,

$$
\inf _{x \in \operatorname{neigh}(0) \cap \mathscr{S}_{\varepsilon}(E)}-\sup _{x \in \operatorname{neigh}(0) \cap \mathscr{U}_{\varepsilon}(E)}=2\left(1+\mathcal{O}\left(\sqrt{\varepsilon}+\alpha^{2}\right)\right)(\varepsilon F)^{\frac{1}{2}} .
$$


In the following, we assume that

$$
E=E_{\varepsilon}-\varepsilon F, \quad \frac{1}{\mathcal{O}(1)} \leq F \leq \mathcal{O}(1),
$$

and we shall define two reference operators $P_{\varepsilon}^{\text {int }}, P_{\varepsilon}^{\text {ext }}$ by "filling the sea" and "filling the well" respectively up to a suitable level. Introduce the metric

$$
\frac{d x^{2}}{\varepsilon+x^{2}}
$$

and let $d_{\varepsilon}$ be the corresponding distance. When (6.23) holds, we see that

$$
d_{\varepsilon}\left(\mathscr{U}_{\varepsilon}(E), \mathscr{S}_{\varepsilon}(E)\right) \asymp 1
$$

If $E<E^{\prime}=E_{\varepsilon}-\varepsilon F^{\prime}, \frac{1}{\mathcal{O}(1)} \leq F^{\prime} \leq \mathcal{O}(1)$, we have

$$
\mathscr{U}_{\varepsilon}\left(E^{\prime}\right) \subset B_{d_{\varepsilon}}\left(\mathscr{U}_{\varepsilon}(E), \mathbf{r}\right), \quad \mathbf{r}=\mathbf{r}\left(E, E^{\prime}, \varepsilon\right)>0,
$$

where $\mathbf{r} \longrightarrow 0^{+}$when $\frac{E^{\prime}-E}{\varepsilon} \longrightarrow 0$. We have the same inclusions after replacing $\mathscr{U}_{\varepsilon}$ with $\mathscr{S}_{\varepsilon}$.

To $d_{\varepsilon}$ we can associate the symbol classes $S_{\varepsilon}\left(\mathbb{R}^{2 n} ; m\right)$ given by Definition A.5. Precisely, the function $a=a(x)$ independent of $\xi$ belongs to $S_{\varepsilon}\left(\mathbb{R}^{2 n} ; m\right)$ if

$$
\partial^{\alpha} a(x)=\mathcal{O}(1) m(x) R_{\varepsilon}(x)^{-|\alpha|}, \quad \forall \alpha \in \mathbb{N}^{n} .
$$

Here $R_{\varepsilon}$ is given by (A.19) and $0<m \in C^{\infty}\left(\mathbb{R}^{n}\right)$ is an order function independent of $\xi$ (see Definition A.4 (b)).

Lemma 6.1. For every $E^{\prime}=E_{\varepsilon}-\varepsilon F^{\prime}$ with $F-F^{\prime} \asymp 1$ small, we can find $\mathbf{r}>0$, tending to 0 when $F-F^{\prime} \longrightarrow 0$, and $0 \leq W \in C^{\infty}\left(\mathbb{R}^{n}\right)$ such that

$$
\begin{gathered}
W \in S_{\varepsilon}\left(\mathbb{R}^{n} ; r_{\varepsilon}^{2}\right), \\
\operatorname{supp} W \subset B_{d_{\varepsilon}}\left(\mathscr{S}_{\varepsilon}(E), \mathbf{r}\right), \\
V_{\varepsilon}+W \geq E^{\prime}+\frac{r_{\varepsilon}^{2}}{C} \quad \text { in } \mathbb{R}^{n} \backslash B_{d_{\varepsilon}}\left(\mathscr{U}_{\varepsilon}(E), \mathbf{r}\right) .
\end{gathered}
$$

Here the scale function $r_{\varepsilon}$ is given by (A.19).

Proof. This can be done in quite a standard way, using partitions of unity, adapted to the metric. See e.g. [11, Remarque 1.3, p. 9]. 
Put

$$
P_{\varepsilon}^{\mathrm{int}}=P_{\varepsilon}+W, \quad V_{\varepsilon}^{\mathrm{int}}=V_{\varepsilon}+W .
$$

We next turn to the definition of $P_{\varepsilon}^{\text {ext }}$ by means of "filling the well". For technical reasons, we want the perturbation to be of trace class.

The inequality $e^{-t}+t \geq 1$ for $t \geq 0$ implies that

$$
\beta e^{-\frac{\xi^{2}}{2 \beta}}+\frac{\xi^{2}}{2} \geq \beta
$$

for every $\beta>0$.

Lemma 6.2. For every $E^{\prime}=E_{\varepsilon}-\varepsilon F^{\prime}$ with $F-F^{\prime} \asymp 1$ small, we can find $\mathbf{r}>0$, tending to 0 when $F-F^{\prime} \longrightarrow 0$, and $0 \leq \beta \in C_{0}^{\infty}\left(\mathbb{R}^{n}\right)$ such that

$$
\begin{gathered}
\beta \in S_{\varepsilon}\left(\mathbb{R}^{2 n} ; r_{\varepsilon}^{2}\right), \\
\operatorname{supp} \beta \subset B_{d_{\varepsilon}}\left(\mathscr{U}_{\varepsilon}(E), \mathbf{r}\right), \\
V_{\varepsilon}+\beta \geq E^{\prime} \quad \text { in } \mathbb{R}^{n} \backslash B_{d_{\varepsilon}}\left(\mathscr{S}_{\varepsilon}(E), \mathbf{r}\right) .
\end{gathered}
$$

Proof. This can be done in quite a standard way, using partitions of unity, adapted to the metric. See e.g. [11, Remarque 1.3, p. 9].

From (6.32), (6.35) we see that

$$
p_{\varepsilon}(x, \xi)+\beta(x) e^{-\frac{\xi^{2}}{2 \beta(x)}} \geq \frac{\xi^{2}}{2}+V_{\varepsilon}(x)+\beta(x) \geq E^{\prime}
$$

in $\mathbb{R}^{n} \backslash B_{d_{\varepsilon}}\left(\mathscr{S}_{\varepsilon}(E), \mathbf{r}\right)$.

We can arrange so that

$$
\beta \geq \frac{r_{\varepsilon}^{2}}{\mathcal{O}(1)} \quad \text { in } B_{d_{\varepsilon}}\left(\mathscr{U}_{\varepsilon}(E), \frac{3 \mathbf{r}}{4}\right)
$$

and

$$
V_{\varepsilon} \geq E^{\prime} \quad \text { in } B_{d_{\varepsilon}}\left(\mathscr{U}_{\varepsilon}(E), \mathbf{r}\right) \backslash B_{d_{\varepsilon}}\left(\mathscr{U}_{\varepsilon}(E), \frac{\mathbf{r}}{2}\right)
$$

Let $\chi_{\mathscr{U}_{\varepsilon}} \in C_{0}^{\infty}\left(B_{d_{\varepsilon}}\left(\mathscr{U}_{\varepsilon}(E), \frac{3 \mathbf{r}}{4}\right) ;[0,1]\right)$ be of class $S_{\varepsilon}\left(\mathbb{R}^{n} ; 1\right)$ and equal to one on $B_{d_{\varepsilon}}\left(\mathscr{U}_{\varepsilon}(E), \frac{\mathbf{r}}{2}\right)$.

Proposition 6.3. We have

$$
\beta \exp \left(-\frac{\xi^{2}}{2 \beta}\right) \in S_{\varepsilon}\left(B_{d_{\varepsilon}}\left(\mathscr{U}_{\varepsilon}(E), \frac{3 \mathbf{r}}{4}\right) \times \mathbb{R}^{n} ; r_{\varepsilon}^{2}\right) .
$$

Here $S_{\varepsilon}(\bullet ; m)$ is given in Definition A.5 (c). 
Proof. We write

$$
\exp \left(-\frac{\xi^{2}}{2 \beta(x)}\right)=\exp \left(-\frac{\tilde{r}_{\varepsilon}^{2}}{2 \beta}\right) \exp \left(\frac{r_{\varepsilon}^{2}}{2 \beta}\right) .
$$

Here $\frac{r_{\varepsilon}^{2}}{2 \beta} \asymp 1$ so $\exp \left(\frac{r_{\varepsilon}^{2}}{2 \beta}\right) \in S_{\varepsilon}(1)$. This factor does not depend on $\xi$. As for the first factor in (6.39), we notice that

$$
0<\frac{\tilde{r}_{\varepsilon}^{2}}{2 \beta} \in S_{\varepsilon}\left(\left(\frac{\tilde{r}_{\varepsilon}}{r_{\varepsilon}}\right)^{2}\right)
$$

is elliptic. For $\alpha, \gamma \in \mathbb{N}^{n}, \partial_{x}^{\alpha} \partial_{\xi}^{\gamma}\left(\exp \left(-\frac{\tilde{r}_{\varepsilon}^{2}}{2 \beta}\right)\right)$ is a finite linear combination of terms

$$
\left(\partial_{x}^{\alpha_{1}} \partial_{\xi}^{\gamma_{1}}\left(\frac{\tilde{r}_{\varepsilon}^{2}}{2 \beta}\right) \ldots \partial_{x}^{\alpha_{k}} \partial_{\xi}^{\gamma_{k}}\left(\frac{\tilde{r}_{\varepsilon}^{2}}{2 \beta}\right)\right) \exp \left(-\frac{\tilde{r}_{\varepsilon}^{2}}{2 \beta}\right)
$$

with $\alpha_{1}+\ldots+\alpha_{k}=\alpha, \gamma_{1}+\ldots+\gamma_{k}=\gamma,\left(\alpha_{k}, \gamma_{k}\right) \neq(0,0)$. This term is

$$
=\mathcal{O}(1)\left(\frac{\tilde{r}_{\varepsilon}^{2}}{\beta}\right)^{k} \exp \left(-\frac{\tilde{r}_{\varepsilon}^{2}}{2 \beta}\right) \tilde{r}_{\varepsilon}^{-|\gamma|} R_{\varepsilon}^{-|\alpha|}=\mathcal{O}(1) \tilde{r}_{\varepsilon}^{-|\gamma|} R_{\varepsilon}^{-|\alpha|}
$$

so $\exp \left(-\frac{\tilde{r}_{\varepsilon}^{2}}{2 \beta}\right) \in S_{\varepsilon}(1)$ and the proposition follows.

Using that $\exp \left(-\frac{\tilde{r}_{\varepsilon}^{2}}{2 \beta}\right)=\mathcal{O}\left(\left(\frac{\tilde{r}_{\varepsilon}}{R_{\varepsilon}}\right)^{-N}\right)$ for every $N \geq 0$, we can strengthen the conclusion in the proposition to

$$
\beta \exp \left(-\frac{\xi^{2}}{2 \beta}\right) \in S_{\varepsilon}\left(B_{d_{\varepsilon}}\left(\mathscr{U}_{\varepsilon}(E), \frac{3 \mathbf{r}}{4}\right) \times \mathbb{R}^{n} ; r_{\varepsilon}^{2}\left(\frac{r_{\varepsilon}}{\tilde{r}_{\varepsilon}}\right)^{N}\right),
$$

for every $N \geq 0$. Using also the properties of $\chi_{\mathscr{U}_{\varepsilon}}$, we get

$$
\chi_{\mathscr{U}_{\varepsilon}}^{2} \beta \exp \left(-\frac{\xi^{2}}{2 \beta}\right) \in S_{\varepsilon}\left(\mathbb{R}^{2 n} ; r_{\varepsilon}^{2}\left(\frac{r_{\varepsilon}}{\tilde{r}_{\varepsilon}}\right)^{N}\right), \quad \forall N \geq 0 .
$$

Here $R_{\varepsilon} \asymp r_{\varepsilon}$ over supp $\chi_{\mathscr{U}_{\varepsilon}}$. We define

$$
p_{\varepsilon}^{\operatorname{ext}}(x, \xi)=p_{\varepsilon}(x, \xi)+\chi_{\mathscr{U}_{\varepsilon}}(x)^{2} \beta(x) \exp \left(-\frac{\xi^{2}}{2 \beta(x)}\right) .
$$

By direct checking,

$$
p_{\varepsilon} \in S_{\varepsilon}\left(\Lambda_{G} ; \tilde{r}_{\varepsilon}^{2}\right)
$$

and by using also (6.43),

$$
p_{\varepsilon}^{\text {ext }} \in S_{\varepsilon}\left(\Lambda_{G} ; \tilde{r}_{\varepsilon}^{2}\right) .
$$




\section{Study of $P_{\varepsilon}^{\text {int }}$}

Recall the definition of $P_{\varepsilon}^{\text {int }}$ in (6.31) and Lemma 6.1, where $E$ is chosen as in (6.23). We make the assumption (A.24):

$$
\varepsilon \geq h^{\frac{1}{2}-\alpha_{0}} \quad \text { for some fixed } \alpha_{0}>0 .
$$

With $E^{\prime}<E, \frac{\varepsilon}{\mathcal{O}(1)} \leq E-E^{\prime} \ll \varepsilon$ as in Lemma 6.1 , we know that the self-adjoint operator $P_{\varepsilon}^{\text {int }}$ has purely discrete spectrum in $]-\infty, E^{\prime}[$.

Recall here that 0 is a non-degenerate saddle point for $V_{\varepsilon}$, with critical value $E_{\varepsilon}=\varepsilon \chi(0,0)$ (cf. (6.11)). From (6.8) we see that $(0,0)$ is a non-degenerate saddle point of $p_{\varepsilon}(x, \xi)=p(x, \xi)+\chi_{\varepsilon}(x, \xi)$ with the same critical value $E_{\varepsilon}$. The discussion of wells and seas in Section 6 can be lifted in a straight forward way from $\mathbb{R}^{n}$ to $T^{*} \mathbb{R}^{n}$. For $-\mathcal{O}(\varepsilon) \leq \widetilde{E} \leq E_{\varepsilon}$ we have (cf. (6.18) that

$$
p_{\varepsilon}^{-1}(]-\infty, \widetilde{E}[)=\widehat{\mathscr{U}}_{\varepsilon}(\widetilde{E}) \cup \widehat{\mathscr{S}}_{\varepsilon}(\widetilde{E}),
$$

where $\widehat{\mathscr{U}}_{\varepsilon}(\widetilde{E}), \widehat{\mathscr{S}}_{\varepsilon}(\widetilde{E})$ are open, $\widehat{\mathscr{U}}_{\varepsilon}(\widetilde{E})$ is bounded and (cf. (6.18))

$$
\pi_{x}\left(\widehat{\mathscr{U}}_{\varepsilon}(\widetilde{E})\right)=\mathscr{U}_{\varepsilon}(\widetilde{E}), \quad \pi_{x}\left(\widehat{\mathscr{S}}_{\varepsilon}(\widetilde{E})\right)=\mathscr{S}_{\varepsilon}(\widetilde{E})
$$

Here $\pi_{x}: T^{*} \mathbb{R}^{n} \longrightarrow \mathbb{R}^{n}$ is the standard base space projection. We write

$$
\widehat{\mathscr{U}}_{\varepsilon}=\widehat{\mathscr{U}}_{\varepsilon}\left(E_{\varepsilon}\right), \quad \widehat{\mathscr{S}}_{\varepsilon}=\widehat{\mathscr{S}}_{\varepsilon}\left(E_{\varepsilon}\right) \text {. }
$$

When $E^{\prime}$ comes close to $E$, we know by Lemma 6.1 that $\operatorname{supp} W$ is close to $\mathscr{S}_{\varepsilon}(E)$ and in particular disjoint from $\pi_{x} \overline{\widehat{\mathscr{U}}}_{\varepsilon}=\overline{\mathscr{U}}_{\varepsilon}$. (Cf. (6.12).)

The eigenvalues of $P_{\varepsilon}^{\text {int }}$ distribute according to the semi-classical Weyl law:

Proposition 7.1. For every fixed $0<\varepsilon \ll 1$ and $-\mathcal{O}(\varepsilon) \leq a<b \leq E$, we have

$$
\#\left(\sigma\left(P_{\varepsilon}^{\mathrm{int}}\right) \cap[a, b]\right)=\frac{1}{(2 \pi h)^{n}}\left(\operatorname{vol}\left(p_{\varepsilon}^{-1}([a, b]) \cap \widehat{\mathscr{U}}_{\varepsilon}\right)+\varepsilon o(1)\right),
$$

when $h \longrightarrow 0$, uniformly in $a, b$.

In the remainder of the main text we now abondon (A.24) and adopt the assumption of Theorem 1.1, namely that $0<\delta \ll 1,0<\varepsilon \leq \varepsilon(\delta)$ and $0<h \leq h(\delta, \varepsilon)$.

Since $\varepsilon$ is fixed, this is the standard result. Having no uniformity in $\varepsilon$ we are free to write the remainder as $\varepsilon o(1)$ instead of $o(1)$.

We write

$$
\operatorname{vol}\left(p_{\varepsilon}^{-1}([a, b])\right)=\omega_{\varepsilon}(b)-\omega_{\varepsilon}(a),
$$


where

$$
\left.\left.\omega_{\varepsilon}(a)=\operatorname{vol}\left(p_{\varepsilon}^{-1}(]-\infty, a\right]\right)_{\mid \widehat{\mathscr{U}}_{\varepsilon}}\right) .
$$

In Appendix B, we show that

$$
\omega(a)-\mathcal{O}\left(\varepsilon^{2}\right) \leq \omega_{\varepsilon}(a) \leq \omega(a) .
$$

Here $\omega(E)$ is the $C^{1}$ function already defined by (1.16) or (1.17) when $E \leq 0$, extended to $0<E \ll 1$ by replacing $\mathscr{U}_{0}$ there by the set $\widetilde{\mathscr{U}_{0}}$, defined in Appendix B.

We end the section with some remarks about the resolvent $\left(P_{\varepsilon}^{\text {int }}-z\right)^{-1}$ when

$$
-\mathcal{O}(\varepsilon)<\Re z \leq E, \quad \Im z=\mathcal{O}(\varepsilon),
$$

and $z \notin \sigma\left(P_{\varepsilon}^{\mathrm{int}}\right)$. Recalling (6.38) with $\mathbf{r}$ and $\frac{E^{\prime}-E}{\varepsilon}$ small, we choose the cutoff function $\widetilde{\chi}_{\mathscr{U}_{\varepsilon}} \in C_{0}^{\infty}\left(B_{d_{\varepsilon}}\left(\mathscr{U}_{\varepsilon}(E), \frac{3 \mathbf{r}}{4}\right) ;[0,1]\right)$ of class $S_{\varepsilon}\left(\mathbb{R}^{n} ; r_{\varepsilon}^{2}\right)$ in the sense of Definition A.5 (cf. (6.35) and the slightly different definition of $\chi_{\mathscr{U}_{\varepsilon}}$ after (6.38)) such that

$$
V_{\varepsilon}^{\mathrm{int}}+\tilde{\chi}_{\mathscr{U}_{\varepsilon}}-E^{\prime} \geq \frac{r_{\varepsilon}^{2}}{\mathcal{O}(1)} \quad \text { on } \mathbb{R}^{n} .
$$

The scale $r_{\varepsilon}$ is given by (A.19). Notice that (7.8) remains valid if we increase $E^{\prime}$ by $\frac{\varepsilon}{\mathcal{O}(1)}$. To shorten the notation we write

$$
Q=P_{\varepsilon}^{\mathrm{int}}, \quad \widetilde{Q}=P_{\varepsilon}^{\mathrm{int}}+\widetilde{\chi}_{\mathscr{U}_{\varepsilon}} .
$$

More explicitly (cf. (6.31)),

$$
\widetilde{Q}=-h^{2} \Delta+\left(V+W+\widetilde{\chi}_{\mathscr{U}_{\varepsilon}}\right)(x)+\varepsilon \chi\left(\varepsilon^{-\frac{1}{2}}\left(x, h D_{x}\right)\right),
$$

with symbol

$$
\tilde{q}(x, \xi)=\xi^{2}+\left(V+W+\widetilde{\chi}_{\mathscr{U} \varepsilon}\right)(x)+\varepsilon \chi\left(\frac{x}{\sqrt{\varepsilon}}, \frac{\xi}{\sqrt{\varepsilon}}\right),
$$

belonging to $S_{\varepsilon}\left(\mathbb{R}^{2 n} ; \tilde{r}_{\varepsilon}^{2}\right)$, see (A.19), Definition A.5.

As in Appendix A we put

$$
x=\mu \tilde{x}, \quad \mu=\sqrt{\varepsilon}, \quad h D_{x}=\mu \tilde{h} D_{\tilde{x}}, \quad \tilde{h}=\frac{h}{\mu^{2}},
$$

and get

$$
\frac{1}{\varepsilon} \widetilde{Q}=\left(-\tilde{h}^{2} \Delta_{\tilde{x}}+\chi\left(\tilde{x}, \tilde{h} D_{\tilde{x}}\right)\right)+\frac{1}{\varepsilon}\left(V+W+\widetilde{\chi}_{\mathscr{U}_{\varepsilon}}\right)(\mu \tilde{x}) .
$$

By the sharp Gårding inequality and (6.6) for $\varepsilon=1$, the first of the two terms in the right hand side is $\geq \chi(\tilde{x}, 0)-\mathcal{O}(\tilde{h})$ in the operator sense, hence

$$
\frac{1}{\varepsilon} \widetilde{Q} \geq-\mathcal{O}(\tilde{h})+\frac{1}{\varepsilon} \inf _{x}\left(V_{\varepsilon}(x)+W(x)+\widetilde{\chi}_{\mathscr{U}_{\varepsilon}}(x)\right) \geq \frac{1}{\varepsilon} E^{\prime}-\mathcal{O}(\tilde{h}),
$$


where we used (7.8) in the last step. Thus,

$$
\widetilde{Q} \geq E^{\prime}-\mathcal{O}(h) .
$$

Now restrict the attention to a domain of the form (7.7). From (7.10) we see that the symbol $\tilde{q}(x, \xi)-z$ belongs to $S_{\varepsilon}\left(\mathbb{R}^{2 n} ; \tilde{r}_{\varepsilon}^{2}\right)$ is elliptic in that space. See (A.19) and Definition A.5. Consequently, with $\widetilde{Q}=\tilde{q}\left(\mu\left(\tilde{x}, \tilde{h} D_{\tilde{x}}\right)\right)$, the symbol of $\varepsilon^{-1}(\widetilde{Q}-z)$ is equal to

$$
\tilde{\xi}^{2}+\frac{1}{\varepsilon}\left(V+W+\tilde{\chi}_{\mathscr{U}_{\varepsilon}}\right)(\mu \tilde{x})+\chi(\tilde{x}, \tilde{\xi})-\frac{z}{\varepsilon}
$$

and it is an elliptic element of $S_{\varepsilon, \mu}\left(\mathbb{R}^{2 n} ; \tilde{r}_{\varepsilon, \mu}^{2}\right)$, where the symbol space is defined with respect to the scales $r_{\varepsilon, \mu}(\tilde{x}), R_{\varepsilon, \mu}(\tilde{x}), \tilde{r}_{\varepsilon, \mu}(x, \xi)$ in (A.28). As in [4, Chapter 8, Proposition 8.6] we know that the inverse $\left(\varepsilon^{-1}(\widetilde{Q}-z)\right)^{-1}$ is an $\tilde{h}$-pseudo-differential operator with symbol in the space $S_{1}\left(\mathbb{R}^{2 n} ; \tilde{r}_{\varepsilon, \mu}^{-2}\right)$, where the subscript 1 indicates that we use the constant scales $r=1, R=1$. Back in the original variable, we get $(\widetilde{Q}-$ $z)^{-1}=\mathrm{Op}(r)$ as an $h$-pseudo-differential operator with symbol $r \in \widehat{S}_{\varepsilon, \mu}\left(\mathbb{R}^{2 n} ; \tilde{r}_{\varepsilon}^{-2}\right)$, meaning that

$$
\partial_{x}^{\alpha} \partial_{\xi}^{\beta} r=\mathcal{O}(1) \tilde{r}_{\varepsilon}(x, \xi)^{-2} \mu^{-|\alpha|-|\beta|} .
$$

Also,

$$
r \equiv \frac{1}{\tilde{q}(x, \xi)-z} \bmod \frac{h}{\mu^{2}} \widehat{S}_{\varepsilon, \mu}\left(\mathbb{R}^{2 n} ; \tilde{r}_{\varepsilon}^{-2}\right)
$$

We get exponentially weighted estimates for the resolvent of $\widetilde{Q}$ in the following way: The symbol $\tilde{q}(x, \xi)-z$ can be extended holomorphically in $\xi$ to a $\frac{\sqrt{\varepsilon}}{\mathcal{O}(1)}$-neighborhood of $\mathbb{R}_{\xi}^{n}$, the extended symbol still belongs to $\widehat{S}_{\varepsilon, \mu}\left(\mathbb{R}^{2 n} ; \tilde{r}_{\varepsilon}^{2}\right)$ in the natural sense and it is still elliptic. By the Kuranishi trick we then see that if $f \in C^{\infty}\left(\mathbb{R}^{n}\right)$ is bounded, $|\nabla f| \leq \frac{\sqrt{\varepsilon}}{\mathcal{O}(1)}, \nabla f \in \sqrt{\varepsilon} S_{\varepsilon, \mu}(1)$, then

$$
e^{\frac{f}{h}}(\widetilde{Q}-z) e^{-\frac{f}{h}}=e^{\frac{f}{h}} \widetilde{Q} e^{-\frac{f}{h}}-z
$$

is an elliptic $h$-pseudo-differential operator with symbol in the class $\widehat{S}_{\varepsilon, \mu}\left(\mathbb{R}^{2 n} ; \tilde{r}_{\varepsilon}^{2}\right)$. The inverse is an $h$-pseudo-differential operator with symbol in the class $\widehat{S}_{\varepsilon, \mu}\left(\mathbb{R}^{2 n}\right.$; $\left.\tilde{r}_{\varepsilon}^{-2}\right)$, of norm $\leq \mathcal{O}\left(\frac{1}{\varepsilon}\right)$. Now this inverse is equal to $e^{\frac{f}{h}}(\widetilde{Q}-z)^{-1} e^{-\frac{f}{h}}$, so we conclude that

$$
\begin{aligned}
e^{\frac{f}{h}}(\widetilde{Q}-z)^{-1} e^{-\frac{f}{h}} & =e^{\frac{f}{h}}\left(P_{\varepsilon}^{\text {int }}+\widetilde{\chi}_{\mathscr{U}_{\varepsilon}}-z\right)^{-1} e^{-\frac{f}{h}} \\
& =\mathcal{O}\left(\frac{1}{\varepsilon}\right): L^{2} \longrightarrow L^{2},
\end{aligned}
$$

under the above assumptions on $f$ and $z$.

Similarly, $\widetilde{\chi}_{\mathscr{U}_{\varepsilon}}$ can be viewed as an $h$-pseudo-differential operator with symbol in $\widehat{S}_{\varepsilon, \mu}\left(\mathbb{R}^{2 n} ; r_{\varepsilon}^{2}\right)$ and it follows that $\widetilde{\chi}_{\mathscr{U}_{\varepsilon}}(\widetilde{Q}-z)^{-1}$ and $(\widetilde{Q}-z)^{-1} \widetilde{\chi}_{\mathscr{U}_{\varepsilon}}$ are $h$-pseudo- 
differential operators with symbol in $\widehat{S}_{\varepsilon, \mu}\left(\mathbb{R}^{2 n} ; \frac{r_{\varepsilon}^{2}}{\tilde{r}_{\varepsilon}^{2}}\right) \subset \widehat{S}_{\varepsilon, \mu}\left(\mathbb{R}^{2 n} ; 1\right)$. We conclude that these operators and their conjugations with $\exp \left(\frac{f}{h}\right)$ are $\mathcal{O}(1): L^{2} \longrightarrow L^{2}$.

We next study the resolvent of $Q=P_{\varepsilon}^{\text {int }}$ for $z$ as in (7.7). Assume that $z \notin \sigma\left(P_{\varepsilon}^{\text {int }}\right)$ and let $\delta=\delta(z)$ denote the distance from $z$ to the spectrum. Recall the telescopic formula

$$
\begin{aligned}
(Q-z)^{-1}= & (\widetilde{Q}-z)^{-1}+(\widetilde{Q}-z)^{-1} \widetilde{\chi}_{\mathscr{U}_{\varepsilon}}(\widetilde{Q}-z)^{-1} \\
& +(\widetilde{Q}-z)^{-1} \widetilde{\chi}_{\mathscr{U}_{\varepsilon}}(Q-z)^{-1} \widetilde{\chi}_{\mathscr{U}_{\varepsilon}}(\widetilde{Q}-z)^{-1} .
\end{aligned}
$$

For $f$ as above, assume in addition that

$$
f=\text { Const. } \quad \text { on } \operatorname{supp} \tilde{\chi}_{\mathscr{U}_{\varepsilon}} .
$$

Now $\tilde{\chi}_{\mathscr{U}_{\varepsilon}}=\mathcal{O}(1)$

$$
e^{\frac{f}{h}} \widetilde{\chi}_{\mathscr{U}_{\varepsilon}}(Q-z)^{-1} \tilde{\chi}_{\mathscr{U}_{\varepsilon}} e^{-\frac{f}{h}}=\widetilde{\chi}_{\mathscr{U}_{\varepsilon}}(Q-z)^{-1} \widetilde{\chi}_{\mathscr{U}_{\varepsilon}}=\mathcal{O}\left(\frac{1}{\delta}\right)
$$

and using also (7.13) and the above remark on the composition of $\tilde{\chi}_{\mathscr{U}}$ and the resolvent of $\widetilde{Q}$, we get by conjugating (7.14):

$$
e^{\frac{f}{h}}(Q-z)^{-1} e^{-\frac{f}{h}}=\mathcal{O}\left(\frac{1}{\varepsilon}\right)+\mathcal{O}\left(\frac{1}{\delta}\right)+\mathcal{O}\left(\frac{1}{\delta}\right),
$$

i.e.

$$
e^{\frac{f}{h}}\left(P_{\varepsilon}^{\text {int }}-z\right)^{-1} e^{-\frac{f}{h}}=\mathcal{O}(1) \frac{1}{\delta}: L^{2} \longrightarrow L^{2} .
$$

Summing up, we have:

Proposition 7.2. Let $f=f_{\varepsilon} \in C^{\infty}\left(\mathbb{R}^{n} ; \mathbb{R}\right)$ be bounded with

$$
|\nabla f| \leq \frac{\sqrt{\varepsilon}}{\mathcal{O}(1)}, \quad \nabla f \in \sqrt{\varepsilon} S_{\varepsilon, \mu}\left(\mathbb{R}^{2 n} ; 1\right) .
$$

Then (7.16) holds uniformly for $z$ as in (7.7) with

$$
\operatorname{dist}\left(z, \sigma\left(P_{\varepsilon}^{\mathrm{int}}\right)\right) \geq \delta>0 .
$$

\section{Study of $P_{\varepsilon}^{\text {ext }}$}

We recall the definition of the symbol

$$
p_{\varepsilon}^{\operatorname{ext}}(x, \xi)=p_{\varepsilon}(x, \xi)+\chi_{\mathscr{U}_{\varepsilon}}^{2}(x) \beta(x) e^{-\frac{\xi^{2}}{2 \beta(x)}}
$$

in (6.44). With $R_{\varepsilon}, r_{\varepsilon}, \tilde{r}_{\varepsilon}$ defined in (A.19), we see that

$$
p_{\varepsilon}^{\text {ext }}(x, \xi) \in S_{\varepsilon}\left(\mathbb{R}^{2 n}, \tilde{r}_{\varepsilon}^{2}\right) .
$$


(Notice here that in $(6.43)$, we can replace $R_{\varepsilon}$ by $r_{\varepsilon}$ since $\chi_{\mathscr{U}_{\varepsilon}}$ has compact support and $R_{\varepsilon} \asymp r_{\varepsilon}$ on any fixed compact set.)

From Lemma 6.2, (6.36), (6.37) and (8.1) we see that if $E^{\prime}$ is as in the cited lemma, then

$$
p_{\varepsilon}^{\text {ext }}(x, \xi)-E^{\prime} \geq C^{-1} \tilde{r}_{\varepsilon}^{2}, \quad x \in B_{d_{\varepsilon}}\left(\mathbb{R}^{n} \backslash \mathscr{S}_{\varepsilon}, \mathbf{r}\right),
$$

where $\mathbf{r}$ is as in the lemma. (Strictly speaking, we apply Lemma 6.2 with a slightly increased value $E_{\text {new }}^{\prime}$, where $E_{\text {new }}^{\prime}-E^{\prime} \asymp \frac{\varepsilon}{\mathcal{O}(1)}$, or alternatively we decrease $E^{\prime}$ in this section with $\frac{\varepsilon}{\mathcal{O}(1)}$.) This means that $p_{\varepsilon}^{\text {ext }}-z$ is uniformly elliptic in $S_{\varepsilon}\left(\mathbb{R}^{2 n} ; \tilde{r}_{\varepsilon}^{2}\right)$ when $x$ varies in $B_{d_{\varepsilon}}\left(\mathbb{R}^{2 n} \backslash \mathscr{S}_{\varepsilon}, \mathbf{r}\right)$, uniformly for

$$
z \in]-\mathcal{O}(\varepsilon), E^{\prime}[+i]-\mathcal{O}(\varepsilon), \mathcal{O}(\varepsilon)[.
$$

Let $G, \widetilde{G}$ be the escape functions in Section 5 and recall that $\widetilde{G}$ is an extension of $G$ from a small neighborhood of $(0,0)$. For simplicity, we drop the tilde in the following, so that $G$ now denotes the globally defined escape function. With $\mathbf{r}$ as above, we may arrange so that with $\mathscr{U}_{\varepsilon}, \mathscr{S}_{\varepsilon}$ defined after (6.18),

$$
\pi_{x}(\operatorname{supp} G) \subset B_{d_{\varepsilon}}\left(\mathbb{R}^{n} \backslash \mathscr{S}_{\varepsilon}, \mathbf{r}\right) .
$$

From Proposition 5.2 and (5.3) we conclude that for $t>0$ small enough,

$$
p_{\varepsilon}^{\operatorname{ext}}\left(\rho+i t H_{G}(\rho)\right)-z \in S_{\varepsilon}\left(\mathbb{R}^{2 n} ; \tilde{r}_{\varepsilon}^{2}\right)
$$

is a uniformly elliptic symbol on $\mathbb{R}^{2 n}$ for

$$
-\mathcal{O}(\varepsilon)<\Re z<E^{\prime}, \quad-\frac{t \varepsilon}{\mathcal{O}(1)}<\Im z<\mathcal{O}(\varepsilon) .
$$

Here we replace $E^{\prime}$ by $\min \left(E^{\prime}, b \varepsilon\right)$ where $b$ is given in Proposition 5.2. (In the end we will have $E^{\prime} \asymp \frac{\varepsilon}{\mathcal{O}(1)}$.)

We now apply Appendix A. Let $G_{\mu}(\widetilde{\alpha})=\mu^{-2} G(\mu \widetilde{\alpha})$. Recalling that $\Lambda_{t G}$ is defined by $\Im \alpha=t H_{G}(\Re \alpha)$, we define $\Lambda_{t G_{\mu}}$ similarly by $\Im \widetilde{\alpha}=t H_{G_{\mu}}(\Re \widetilde{\alpha})$. More explicitly, the latter manifold is given by

$$
\Im \widetilde{\alpha}_{x}=t \partial_{\widetilde{\alpha}_{\xi}} G_{\mu}(\Re \widetilde{\alpha}), \quad \Im \widetilde{\alpha}_{\xi}=-t \partial_{\widetilde{\alpha}_{x}} G_{\mu}(\Re \widetilde{\alpha}) .
$$

Putting $\alpha=\mu \widetilde{\alpha}$, we get $\alpha \in \Lambda_{t G}$. Thus we have the bijection

$$
\Lambda_{t G_{\mu}} \ni \widetilde{\alpha} \longmapsto \mu \widetilde{\alpha} \in \Lambda_{t G} .
$$

In Appendix A, we defined the FBI-transformations $T, T_{\mu}$ and noted that

$$
T_{\mu} \tilde{u}(\widetilde{\alpha} ; \tilde{h})=T u(\alpha ; h) \quad \text { with } \quad u(x)=\mu^{\frac{n}{2}} \tilde{u}(\tilde{x})
$$

for $\alpha \in \Lambda_{t G}, \widetilde{\alpha} \in \Lambda_{t G_{\mu}}$ related by $\alpha=\mu \widetilde{\alpha}$. See (A.32)-(A.38). 
We can define the spaces $H\left(\Lambda_{t G_{\mu}} ; m_{\mu}\right)$ as in [11, Chapter 5] and define the space $H\left(\Lambda_{t G} ; m\right)$ by requiring that $(\mathrm{A} .41)$ holds when $m_{\mu}(\widetilde{\alpha})=m(\mu \widetilde{\alpha})$.

We define

$$
P_{\varepsilon}^{\mathrm{ext}}:=P_{\varepsilon}+\chi_{\mathscr{U}_{\varepsilon}} \mathrm{Op}_{h}\left(\beta e^{-\frac{\xi^{2}}{2 \beta}}\right) \chi_{\mathscr{U}_{\varepsilon}},
$$

where $\mathrm{Op}_{h}$ denotes the $h$-Weyl quantization. We view $P_{\varepsilon}^{\text {ext }}$ as an $h$-quantization of $p_{\varepsilon}^{\text {ext }}$ in (8.1).

The scaling $x=\mu \tilde{x}$ transforms the operator $\varepsilon^{-1}\left(P_{\varepsilon}^{\text {ext }}-z\right)$ into an $\tilde{h}$-pseudodifferential operator of class $S\left(\Lambda_{t G_{\mu}} ; \tilde{r}_{\varepsilon, \mu}^{2}\right)$ which is uniformly elliptic on $\Lambda_{t G_{\mu}}$ when $t>0$ is small and fixed and $z$ varies in the set (8.4). Consequently, this operator is bijective with a uniformly bounded inverse $H\left(\Lambda_{t G_{\mu}} ; \tilde{r}_{\varepsilon, \mu}^{2}\right) \longrightarrow H\left(\Lambda_{t G_{\mu}}\right)$. This means that

$$
P_{\varepsilon}^{\mathrm{ext}}-z: H\left(\Lambda_{t G} ; \tilde{r}_{\varepsilon}^{2}\right) \longrightarrow H\left(\Lambda_{t G}\right)
$$

is bijective with a uniformly bounded inverse for $z$ in the set (8.4). Since $\tilde{r}_{\varepsilon}^{2} \geq \varepsilon$, it follows that for $z$ in the same set,

$$
\left(P_{\varepsilon}^{\mathrm{ext}}-z\right)^{-1}=\left\{\begin{array}{l}
\mathcal{O}(1): H\left(\Lambda_{t G}\right) \longrightarrow H\left(\Lambda_{t G}, \tilde{r}_{\varepsilon}^{2}\right), \\
\mathcal{O}\left(\frac{1}{\varepsilon}\right): H\left(\Lambda_{t G}\right) \longrightarrow H\left(\Lambda_{t G}\right) .
\end{array}\right.
$$

\section{Study of $P_{\varepsilon}$}

We will incorporate ordinary exponentially weighted estimates in the machinery of [11] and recall from Chapter 5 in that work that the spaces $H\left(\Lambda_{G} ; m\right)$ can be defined when $G(\alpha)-g\left(\alpha_{x}\right)$ is sufficiently small in $S(r R)$ and $g-g_{0}\left(\alpha_{x}\right)$ is sufficiently small in $\dot{S}^{1,1}\left(\mathbb{R}^{n}\right)=\dot{S}\left(\mathbb{R}^{n} ; R \tilde{r}\right)$ and we work with a fixed FBI transform, depending only on $g_{0}$. Moreover, when $G=g\left(\alpha_{x}\right)$ is independent of $\alpha_{\xi}$, then

$$
H\left(\Lambda_{g}\right):=H\left(\Lambda_{g} ; 1\right)=L^{2}\left(\Lambda_{g} ; e^{-\frac{2}{h} g(x)} d x\right) .
$$

(See [11, Proposition 5.3].) When replacing the weight 1 with suitable weights (like a power of $\tilde{r}$ ) we get the corresponding naturally defined Sobolev spaces.

According to [11, Proposition 5.7], if $\widetilde{G}$ is a second function with the same structure as $G$ and with the same basic weight $g_{0}$, and if $\Lambda_{G} \leq \Lambda_{\widetilde{G}}$ in the sense that $G \leq \widetilde{G}$ and if $\widetilde{m}$ is a second order function with $m \geq \widetilde{m}$, then

$$
H\left(\Lambda_{G} ; m\right) \subset H\left(\Lambda_{\widetilde{G}} ; \widetilde{m}\right)
$$

and the inclusion map is uniformly bounded. For more details see Appendix A. 
This theory is based on the use of scale functions $R, r, \tilde{r}$ satisfying (A.1), (A.2), (A.3), (A.5). However, the dilation in Appendix A allows us to apply it also in the case of the scales $R_{\varepsilon}, r_{\varepsilon}, \tilde{r}_{\varepsilon}$ that do not satisfy (A.5).

From [11, See the proof of Theorem 8.3.], we can partially extend the estimate (7.16). Let $f=f(x)$ be small in the space $\dot{S}\left(\mathbb{R}^{n} ; R_{\varepsilon} r_{\varepsilon}\right)$. Then the theory applies to

$$
\widetilde{Q}:=P_{\varepsilon}^{\mathrm{int}}+\chi_{\mathscr{U}_{\varepsilon}} \mathrm{Op}_{h}\left(\beta e^{-\frac{\xi^{2}}{2 \beta}}\right) \chi_{\mathscr{U}_{\varepsilon}}=: P_{\varepsilon}^{\mathrm{int}}+\widehat{\chi}_{\mathscr{U}_{\varepsilon}},
$$

cf. (8.5), slightly different from " $\widetilde{Q}$ " in (7.9). When $z$ belongs to the set (7.7) for $E=\frac{\varepsilon}{C}$ for $C \gg 1$, we see that

$$
(\widetilde{Q}-z)^{-1}=\mathcal{O}(1):\left\{\begin{aligned}
H\left(\Lambda_{f} ; \tilde{r}_{\varepsilon}^{-2}\right) & \longrightarrow H\left(\Lambda_{f}\right), \\
H\left(\Lambda_{f}\right) & \longrightarrow H\left(\Lambda_{f} ; \tilde{r}_{\varepsilon}^{2}\right) .
\end{aligned}\right.
$$

By the telescopic formula (7.14) for $P_{\varepsilon}^{\text {int }}$, with $\widetilde{\chi}_{\mathscr{U}_{\varepsilon}}$ replaced by $\widehat{\chi}_{\mathscr{U}_{\varepsilon}}$, we see that if (7.15) also holds, then

$$
\left(P_{\varepsilon}^{\mathrm{int}}-z\right)^{-1}-(\widetilde{Q}-z)^{-1}=\mathcal{O}\left(\frac{1}{\delta}\right):\left\{\begin{aligned}
H\left(\Lambda_{f}\right) & \longrightarrow H\left(\Lambda_{f} ; \tilde{r}_{\varepsilon}^{2}\right), \\
H\left(\Lambda_{f} ; \tilde{r}_{\varepsilon}^{-2}\right) & \longrightarrow H\left(\Lambda_{f}\right), \\
H\left(\Lambda_{f}\right) & \longrightarrow H\left(\Lambda_{f}\right) .
\end{aligned}\right.
$$

Since $\tilde{r}_{\varepsilon}^{2} \geq \varepsilon$, the inclusion maps

$$
H\left(\Lambda_{f}, \tilde{r}_{\varepsilon}^{2}\right) \longrightarrow H\left(\Lambda_{f}\right) \text { and } H\left(\Lambda_{f}\right) \longrightarrow H\left(\Lambda_{f}, \tilde{r}_{\varepsilon}^{-2}\right)
$$

have norms $\leq \varepsilon^{-1}$. Estimate (9.3) and the above one for $(\widetilde{Q}-z)^{-1}$ therefore imply that

$$
\left(P_{\varepsilon}^{\mathrm{int}}-z\right)^{-1}=\mathcal{O}\left(\frac{1}{\delta}\right):\left\{\begin{aligned}
H\left(\Lambda_{f}\right) & \longrightarrow H\left(\Lambda_{f} ; \tilde{r}_{\varepsilon}^{2}\right), \\
H\left(\Lambda_{f} ; \tilde{r}_{\varepsilon}^{-2}\right) & \longrightarrow H\left(\Lambda_{f}\right), \\
H\left(\Lambda_{f}\right) & \longrightarrow H\left(\Lambda_{f}\right)
\end{aligned}\right.
$$

We choose $f$ as above with constant 0 in (7.15):

$$
f=0 \text { on } \operatorname{supp} \chi_{\mathscr{U}_{\varepsilon}},
$$

with $f \leq 0$ everywhere and

$$
f \asymp-R_{\varepsilon} r_{\varepsilon} \text { on } \pi_{x}(\operatorname{supp} G) .
$$

This implies that for $t$ small: $\Lambda_{f} \leq \Lambda_{t G}$ and after a further decrease of $t>0$, that

$$
W=\mathcal{O}(1) e^{-\frac{\varepsilon}{\mathcal{O}(h)}}: H\left(\Lambda_{f} ; \tilde{r}_{\varepsilon}^{2}\right) \longrightarrow H\left(\Lambda_{t G}\right) .
$$


Combining this with (9.4), (9.5), we get

$$
W\left(P_{\varepsilon}^{\mathrm{int}}-z\right)^{-1} \widehat{\chi}_{\mathscr{U}_{\varepsilon}}=\mathcal{O}(1) \frac{1}{\delta} e^{-\frac{\varepsilon}{\mathcal{O}(h)}}: H\left(\Lambda_{t G}\right) \longrightarrow H\left(\Lambda_{t G}\right) .
$$

Here we also used that

$$
\widehat{\chi}_{\mathscr{U}_{\varepsilon}}=\mathcal{O}(1): H\left(\Lambda_{f}\right) \longrightarrow H\left(\Lambda_{t G}\right),
$$

in view of $(9.5)$.

In the following, we assume that

$$
\operatorname{dist}\left(z, \sigma\left(P^{\mathrm{int}}\right)\right)=\delta \geq h^{N_{0}}
$$

for some fixed $N_{0}>0$. Then the right hand side in (9.8) can be replaced by $\mathcal{O}(1) e^{-\frac{\varepsilon}{\mathcal{O}(h)}}$.

We can now construct a right inverse of $P_{\varepsilon}-z$. Let $z$ vary in a set of the form (8.4), now with $0<t \ll 1$ fixed and $E^{\prime}=\frac{\varepsilon}{C}$ for a fixed sufficiently large $C \gg 1$. In view of the identity

$$
\left(P_{\varepsilon}-z\right)\left(P_{\varepsilon}^{\mathrm{ext}}-z\right)^{-1}=1-\widehat{\chi}_{\mathscr{U}_{\varepsilon}}\left(P_{\varepsilon}^{\mathrm{ext}}-z\right)^{-1},
$$

we try as an approximate right inverse,

$$
\begin{aligned}
& R_{0}(z)=\left(P_{\varepsilon}^{\mathrm{ext}}-z\right)^{-1}+\left(P_{\varepsilon}^{\mathrm{int}}-z\right)^{-1} \widehat{\chi}_{\mathscr{U}_{\varepsilon}}\left(P_{\varepsilon}^{\mathrm{ext}}-z\right)^{-1} \\
& \quad=\mathcal{O}\left(\frac{1}{\delta}\right): H\left(\Lambda_{t G}\right) \longrightarrow H\left(\Lambda_{t G}, \tilde{r}_{\varepsilon}^{2}\right) \cap H\left(\Lambda_{t G}\right) .
\end{aligned}
$$

We have

$$
\begin{aligned}
\left(P_{\varepsilon}-z\right) R_{0}(z) & =1-K \text { with } \\
K & =W\left(P_{\varepsilon}^{\text {int }}-z\right)^{-1} \widehat{\chi}_{\mathscr{U}_{\varepsilon}}\left(P_{\varepsilon}^{\mathrm{ext}}-z\right)^{-1} .
\end{aligned}
$$

From $(9.8)$, with the right hand side simplified to $\mathcal{O}(1) e^{-\frac{\varepsilon}{\mathcal{O}(h)}}$, we see that

$$
K=\mathcal{O}(1) e^{-\frac{\varepsilon}{\mathcal{O}(h)}}: H\left(\Lambda_{t G}\right) \longrightarrow H\left(\Lambda_{t G}\right) .
$$

for every small fixed $t>0$. Then for $h>0$ small enough, $1-K$ is bijective with inverse $\mathcal{O}(1)$ and we get the right inverse of $P_{\varepsilon}-z$ :

$$
R_{0}(z)(1-K)^{-1}=\mathcal{O}\left(\frac{1}{\delta}\right): H\left(\Lambda_{t G}\right) \longrightarrow H\left(\Lambda_{t G} ; \tilde{r}_{\varepsilon}^{2}\right) \cap H\left(\Lambda_{t G}\right)
$$

From [11, See the end of proof of Theorem 8.3., page 99] we know that $P_{\varepsilon}-z$ : $H\left(\Lambda_{t G} ; \tilde{r}_{\varepsilon}^{2}\right) \longrightarrow H\left(\Lambda_{t G}\right)$ is a Fredholm operator of index 0 so $R_{0}(z)(1-K)^{-1}$ is also a left inverse. 
Proposition 9.1. Let $0<t \ll 1$ and let $z$ vary in $\{z \in \mathbb{C} ;$ (8.4) and (9.9) hold $\}$, where $E^{\prime}=\frac{\varepsilon}{C}, C \gg 1$. Then for $h>0$ small enough, $P_{\varepsilon}-z: H\left(\Lambda_{t G} ; \tilde{r}_{\varepsilon}^{2}\right) \longrightarrow H\left(\Lambda_{t G}\right)$ is bijective and

$$
\left(P_{\varepsilon}-z\right)^{-1}=\mathcal{O}\left(\frac{1}{\delta}\right): H\left(\Lambda_{t G}\right) \longrightarrow H\left(\Lambda_{t G} ; \tilde{r}_{\varepsilon}^{2}\right) \cap H\left(\Lambda_{t G}\right) .
$$

By a variant of the above arguments, we also get:

Proposition 9.2. For $0<t \ll 1$ we restrict the attention to the region (8.4). In this region we have a bijection

$$
b: \sigma\left(P_{\varepsilon}^{\text {int }}\right) \longrightarrow \operatorname{Res}\left(P_{\varepsilon}\right)
$$

such that $b(\mu)-\mu=\mathcal{O}\left(h^{\infty}\right) \cdot\left({ }^{6}\right)$

Proof. It will be convenient to work with a different approximation of $\left(P_{\varepsilon}-\right.$ $z)^{-1}$. Let $\chi_{0} \in C_{0}^{\infty}\left(\mathbb{R}^{n}\right) \cap S_{\varepsilon}(1)$ have the property that for some small fixed $r$ :

$$
\chi_{0}=\left\{\begin{array}{l}
1 \text { on } B_{d_{\varepsilon}}\left(\mathscr{U}_{\varepsilon}, r\right), \\
0 \text { on } B_{d_{\varepsilon}}\left(\mathscr{S}_{\varepsilon}, r\right)
\end{array} .\right.
$$

As a new approximation we take

$$
R_{0}(z)=\left(P_{\varepsilon}^{\mathrm{int}}-z\right)^{-1} \chi_{0}+\left(P_{\varepsilon}^{\mathrm{ext}}-z\right)^{-1}\left(1-\chi_{0}\right),
$$

which satisfies the estimate

$$
R_{0}(z)=\mathcal{O}\left(\frac{1}{\delta}\right): H\left(\Lambda_{t G}\right) \longrightarrow H\left(\Lambda_{t G} ; \tilde{r}_{\varepsilon}^{2}\right) \cap H\left(\Lambda_{t G}\right) .
$$

Then

$$
\begin{aligned}
\left(P_{\varepsilon}-z\right) R_{0}(z) & =1-W\left(P_{\varepsilon}^{\mathrm{int}}-z\right)^{-1} \chi_{0}-\widehat{\chi}_{\mathscr{U}_{\varepsilon}}\left(P_{\varepsilon}^{\mathrm{ext}}-z\right)^{-1}\left(1-\chi_{0}\right) \\
& =: 1-K,
\end{aligned}
$$

where (the new) $K$ satisfies (9.12), if we assume that $\delta \geq h^{N_{0}}$ for some fixed $N_{0}>0$. Then,

$$
\begin{aligned}
\left(P_{\varepsilon}-z\right)^{-1} & =R_{0}(1-K)^{-1}=R_{0}+L \\
& =\mathcal{O}\left(\frac{1}{\delta}\right): H\left(\Lambda_{t G}\right) \longrightarrow H\left(\Lambda_{t G} ; \tilde{r}_{\varepsilon}^{2}\right) \cap H\left(\Lambda_{t G}\right)
\end{aligned}
$$

$\left({ }^{6}\right)$ Strictly speaking, to obtain a bijection, we have to modify the bounds in (8.4) very slightly, so that no point in $\sigma\left(P_{\varepsilon}^{\text {int }}\right) \cup \operatorname{Res}\left(P_{\varepsilon}\right)$ is too close to the boundary of the region $\Omega$ defined by (8.4) and we then get a bijection $b: \Omega \cap \sigma\left(P_{\varepsilon}^{\text {int }}\right) \longrightarrow \Omega \cap \operatorname{Res}\left(P_{\varepsilon}\right)$, when counting the eigenvalues and the resonances with their multiplicity. 
with

$$
L=\mathcal{O}(1) e^{-\frac{\varepsilon}{\mathcal{O}(h)}}: H\left(\Lambda_{t G}\right) \longrightarrow H\left(\Lambda_{t G} ; \tilde{r}_{\varepsilon}^{2}\right)
$$

If $\gamma \subset \Omega$ is a simple closed contour of uniformly bounded length, along which $\delta \geq h^{N_{0}}$, we get from $(9.16),(9.19),(9.20)$ :

$$
\pi_{\varepsilon, \gamma}=\pi_{\varepsilon, \gamma}^{\mathrm{int}} \chi_{0}+\mathcal{O}(1) e^{-\frac{\varepsilon}{\mathcal{O}(h)}}: H\left(\Lambda_{t G}\right) \longrightarrow H\left(\Lambda_{t G}\right),
$$

where

$$
\begin{aligned}
& \pi_{\varepsilon, \gamma}=\frac{1}{2 \pi i} \int_{\gamma}\left(z-P_{\varepsilon}\right)^{-1} d z, \\
& \pi_{\varepsilon, \gamma}^{\text {int }}=\frac{1}{2 \pi i} \int_{\gamma}\left(z-P_{\varepsilon}^{\text {int }}\right)^{-1} d z,
\end{aligned}
$$

are the spectral projections of $P_{\varepsilon}, P_{\varepsilon}^{\text {int }}$ respectively, associated to the part of the spectra inside $\gamma$. By exponentially weighted estimates,

$$
\pi_{\varepsilon, \gamma}^{\text {int }} \chi_{0}-\pi_{\varepsilon, \gamma}^{\text {int }}=\mathcal{O}(1) e^{-\frac{\varepsilon}{\mathcal{O}(h)}}
$$

and we conclude that

$$
\operatorname{rank} \pi_{\varepsilon, \gamma}=\operatorname{rank} \pi_{\varepsilon, \gamma}^{\mathrm{int}} \text {. }
$$

Hence $P_{\varepsilon}$ and $P_{\varepsilon}^{\text {int }}$ have the same number of eigenvalues inside $\gamma$. Varying $\gamma$ and $N_{0}$, we get the proposition.

\section{Resolvents of other operators}

We start with the resolvent of $P$ that we realize as an operator from $H\left(\Lambda_{t G} ; \tilde{r}_{\varepsilon}^{2}\right)$ to $H\left(\Lambda_{t G}\right)$ with the same $G$ as above. Then $P$ has discrete spectrum in the set $(8.4)$ and the eigenvalues are confined to the lower half plane. They are the resonances that we want to study. Restricting now the attention to the set

$$
-\mathcal{O}(\varepsilon)<\Re z<-\frac{\varepsilon}{C}, \quad-\frac{t}{C} \varepsilon<\Im z<\mathcal{O}(\varepsilon),
$$

where $C \gg 1$ is large enough, we can adapt the discussion for $P_{\varepsilon}$ to $P$. Using Proposition 5.1 rather than Proposition 5.2, we get

Proposition 10.1. Let $0<t \ll 1$ and let $z$ vary in the set (10.1). If $|\Im z| \geq \delta \geq$ $h^{N_{0}}$ for some fixed $N_{0}>0$, then $P-z: H\left(\Lambda_{t G} ; \tilde{r}_{\varepsilon}^{2}\right) \longrightarrow H\left(\Lambda_{t G}\right)$ is bijective and

$$
(P-z)^{-1}=\mathcal{O}\left(\frac{1}{\delta}\right): H\left(\Lambda_{t G}\right) \longrightarrow H\left(\Lambda_{t G} ; \tilde{r}_{\varepsilon}^{2}\right) \cap H\left(\Lambda_{t G}\right)
$$

In addition to $P_{\varepsilon}$ we need a reference operator with two gaps in the spectrum near $\mathbb{R}$. Recall that $P_{\varepsilon}^{\text {int }}$ has discrete spectrum in $]-\mathcal{O}(\varepsilon), E\left[, E=\frac{\varepsilon}{C}\right.$, and that we 
have Weyl asymptotics there by Proposition 7.1. In particular,

$$
\#\left(\sigma\left(P_{\varepsilon}^{\mathrm{int}}\right) \cap(A \varepsilon+]-\frac{\delta \varepsilon}{2}, \frac{\delta \varepsilon}{2}[)\right)=\mathcal{O}\left(\delta \varepsilon h^{-n}\right),
$$

uniformly when $\varepsilon \leq \varepsilon(\delta), h \leq h(\varepsilon, \delta)$ ( $t$ fixed) and $A$ varies in the interval defined by $-\mathcal{O}(\varepsilon)+\frac{\delta \varepsilon}{2} \leq A \varepsilon \leq E-\frac{\delta \varepsilon}{2}$.

Let $\mu_{1}, \ldots, \mu_{N}\left(\right.$ with $\left.N=\mathcal{O}\left(\delta \varepsilon h^{-n}\right)\right)$ be the eigenvalues of $P_{\varepsilon}^{\text {int }}$ in $\left.A \varepsilon+\right]-\frac{\delta \varepsilon}{2}, \frac{\delta \varepsilon}{2}[$ and let $e_{1}, \ldots, e_{N} \in L^{2}\left(\mathbb{R}^{n}\right)$ be a corresponding orthonormal family of eigenfunctions, so that

$$
\mathbf{1}_{A \varepsilon+]-\frac{\delta \varepsilon}{2}, \frac{\delta \varepsilon}{2}[}\left(P_{\varepsilon}^{\mathrm{int}}\right) u=\sum_{j} \mu_{j}\left(u \mid e_{j}\right) e_{j},
$$

where $(\cdot \mid \cdot)$ is the usual inner product in $L^{2}\left(\mathbb{R}^{n}\right)$. We create a gap in the spectrum by moving each $\mu_{j}$ to the closest of the two boundary points $A \varepsilon-\frac{\delta \varepsilon}{2}, A \varepsilon+\frac{\delta \varepsilon}{2}$ : Put

$$
\tilde{\mu}_{j}=\left\{\begin{array}{l}
A \varepsilon-\frac{\delta \varepsilon}{2} \text { if } \mu_{j} \leq A \varepsilon \\
A \varepsilon+\frac{\delta \varepsilon}{2} \text { if } \mu_{j}>A \varepsilon
\end{array}\right.
$$

and set

$$
\widetilde{P}_{\varepsilon, A, \delta}^{\mathrm{int}} u=P_{\varepsilon}^{\mathrm{int}} u+\sum_{j}\left(\tilde{\mu}_{j}-\mu_{j}\right)\left(u \mid e_{j}\right) e_{j},
$$

so that the eigenvalues $\mu_{j}$ of $P_{\varepsilon}^{\text {int }}$ become the eigenvalues $\tilde{\mu}_{j}$ of $\widetilde{P}_{\varepsilon, A, \delta}^{\text {int }}$ while the eigenvalues of $P_{\varepsilon}^{\text {int }}$ outside $\left.A \varepsilon+\right]-\frac{\delta \varepsilon}{2}, \frac{\delta \varepsilon}{2}$ [ remain unchanged.

Now we know that $e_{j}$ decay exponentially outside $\mathscr{U}_{\varepsilon}$, as shown in the discussion around (9.4), so if $\chi_{\mathscr{U}_{\varepsilon}}$ is the cutoff function in (6.43), (8.1), (8.5), then

$$
\left\|\widetilde{P}_{\varepsilon, A, \delta}^{\text {int }}-P_{\varepsilon, A, \delta}^{\text {int }}\right\|_{\operatorname{tr}}=\mathcal{O}(1) e^{-\frac{\varepsilon}{\mathcal{O}(h)}},
$$

where

$$
P_{\varepsilon, A, \delta}^{\mathrm{int}} \bullet=P_{\varepsilon}^{\mathrm{int}} \bullet+\chi_{\mathscr{U}_{\varepsilon}} \sum_{j}\left(\tilde{\mu}_{j}-\mu_{j}\right)\left(\chi_{\mathscr{U}_{\varepsilon}} \bullet \mid e_{j}\right) e_{j} .
$$

From (10.6) it follows that

$$
\sigma\left(P_{\varepsilon, A, \delta}^{\mathrm{int}}\right) \cap\left(A \varepsilon+\left[-\frac{\varepsilon \delta}{3}, \frac{\varepsilon \delta}{3}\right]\right)=\varnothing .
$$

Here " 3 " can be replaced by any number $>2$.

Notice that we could have replaced the definition of $P_{\varepsilon}^{\text {ext }}$ in (8.5) by

$$
P_{\varepsilon}^{\mathrm{ext}} \bullet=P_{\varepsilon} \bullet+\chi \chi_{\varepsilon} \sum_{\mu \in]-\infty, E\left[\cap \sigma\left(P_{\varepsilon}^{\mathrm{int}}\right)\right.}(E-\mu)\left(\chi_{\mathscr{U}_{\varepsilon}} \bullet \mid e_{\mu}\right) e_{\mu},
$$


where $e_{\mu}$ denotes the orthonormal system of eigenfunctions associated to the $\mu \in$ ]$-\infty, E\left[\cap \sigma\left(P_{\varepsilon}^{\text {int }}\right)\right.$.

Now, put

$$
P_{\varepsilon, A, \delta} \bullet=P_{\varepsilon} \bullet+\chi_{\mathscr{U} \varepsilon} \sum_{j}\left(\tilde{\mu}_{j}-\mu_{j}\right)\left(\chi_{\mathscr{U}_{\varepsilon}} \bullet \mid e_{j}\right) e_{j},
$$

acting on $H\left(\Lambda_{t G} ; \tilde{r}_{\varepsilon}^{2}\right)$. As in Section 9 , if we restrict the attention to the region (10.1), there is a bijection

$$
b: \sigma\left(P_{\varepsilon, A, \delta}^{\mathrm{int}}\right) \longrightarrow \operatorname{Res}\left(P_{\varepsilon, A, \delta}\right),
$$

such that $b(\mu)-\mu=\mathcal{O}\left(h^{\infty}\right)$ (with the same proviso as in the footnote to Proposition 9.2). In particular, for every fixed $N_{0}>0, P_{\varepsilon, A, \delta}$ has no resonances outside an $h^{N_{0}}$-neighborhood of

$$
]-\mathcal{O}(\varepsilon), E^{\prime}\left[\backslash(A \varepsilon+]-\frac{\varepsilon \delta}{2}, \frac{\varepsilon \delta}{2}[)\right.
$$

when $h$ is small. Outside such a neighborhood in the set (10.1), we have

$$
\left\|\left(P_{\varepsilon, A, \delta}-z\right)^{-1}\right\| \leq \mathcal{O}(1)\left(\operatorname{dist}\left(z, \mathbb{R} \backslash(A \varepsilon+]-\frac{\varepsilon \delta}{2}, \frac{\varepsilon \delta}{2}[)\right)\right)^{-1}
$$

as a bounded operator in $H\left(\Lambda_{t G}\right)$.

In the same way, we can build reference operators with two gaps. Let $B \in \mathbb{R}$ be a second energy level as in (10.3) and assume in addition that $B-A \geq \delta$. We first define $\widetilde{P}_{\varepsilon, A, B, \delta}^{\text {int }}$ as in (10.5) by replacing each eigenvalue $\mu_{j}$ of $P_{\varepsilon}^{\text {int }}$ in $\left.\{A \varepsilon, B \varepsilon\}+\right]-\frac{\delta \varepsilon}{2}, \frac{\delta \varepsilon}{2}[$ by the closest boundary point $\tilde{\mu}_{j}$ of this set. Then we define $P_{\varepsilon, A, B, \delta}^{\text {int }}$ as in (10.7). We have the obvious modifications of the bijection in (10.10) and the resolvent estimate (10.11). Let us also notice that

$$
\begin{aligned}
\#\left(\operatorname{Res}\left(P_{\varepsilon, A, B, \delta}\right)\right. & \left.\cap(] A \varepsilon, B \varepsilon[+i]-\frac{\varepsilon}{\mathcal{O}(1)}, \mathcal{O}(\varepsilon)[)\right) \\
& =\#\left(\sigma\left(P_{\varepsilon, A, B, \delta}^{\mathrm{int}}\right) \cap\right] A \varepsilon, B \varepsilon[) \\
& =(\omega(\varepsilon B)-\omega(\varepsilon A))(2 \pi h)^{-n}+\mathcal{O}(\delta \varepsilon) h^{-n},
\end{aligned}
$$

where the volume function $\omega$ is discussed in Appendix B. This estimate is uniform for $0<\delta \ll 1,0<\varepsilon \leq \varepsilon(\delta), 0<h \leq h(\delta, \varepsilon)$. $(0<t \ll 1$ is fixed.)

\section{Relative determinants}

Fix $t>0$ small so that the earlier estimates are valid in

$$
\left.R=R_{C_{1}, C}(\varepsilon)=\right]-\mathcal{O}(\varepsilon), \frac{\varepsilon}{C}[+i]-\frac{\varepsilon}{C_{1}}, \mathcal{O}(\varepsilon)[
$$


or in certain explicitly given subsets of this region. Here $C, \mathcal{O}(\varepsilon)$ are as in $(10.1)$ and $C_{1}>0$ is large enough, depending on $t .\left({ }^{7}\right)$

In (8.7) we have seen that uniformly for $z \in R$,

$$
\left(P_{\varepsilon}^{\mathrm{ext}}-z\right)^{-1}=\left\{\begin{array}{c}
\mathcal{O}(1): H\left(\Lambda_{t G}\right) \longrightarrow H\left(\Lambda_{t G} ; \tilde{r}_{\varepsilon}^{2}\right), \\
\mathcal{O}\left(\frac{1}{\varepsilon}\right): H\left(\Lambda_{t G}\right) \longrightarrow H\left(\Lambda_{t G}\right) .
\end{array}\right.
$$

From the definition of $P_{\varepsilon}$ in the beginning of Section 6 we see that

$$
\left\|P-P_{\varepsilon}\right\|_{\mathrm{tr}}=\mathcal{O}(1) \varepsilon\left(\frac{\varepsilon}{h}\right)^{n}
$$

In fact, from (4.3) we see that the $h$-quantization of $\chi_{\varepsilon}$ is unitarily equivalent to the $h=1$-quantization of $a_{\varepsilon, h}(x, \xi)=\varepsilon \chi\left(\sqrt{\frac{h}{\varepsilon}}(x, \xi)\right)$. Recalling that $\chi \in \mathcal{S}$ and that $0<\frac{h}{\varepsilon} \leq 1$, we have

$$
\sum_{|\alpha| \leq 2 n+1}\left\|\partial_{x, \xi}^{\alpha} a_{\varepsilon, h}\right\|_{L^{1}\left(\mathbb{R}^{2 n}\right)} \leq \mathcal{O}\left(\varepsilon\left(\frac{\varepsilon}{h}\right)^{n}\right)
$$

and applying for instance [4, Theorem 9.4], we get $\left\|\chi_{\varepsilon}\left(x, h D_{x}\right)\right\|_{\operatorname{tr}} \leq \mathcal{O}\left(\varepsilon\left(\frac{\varepsilon}{h}\right)^{n}\right)$.

Similarly from the definition of $P_{\varepsilon}^{\text {ext }}$ in (8.1), (8.5), we have

$$
\left\|P_{\varepsilon}-P_{\varepsilon}^{\mathrm{ext}}\right\|_{\mathrm{tr}}=\mathcal{O}(1) h^{-n}
$$

Thus,

$$
\left\|P-P_{\varepsilon}^{\mathrm{ext}}\right\|_{\mathrm{tr}}=\mathcal{O}(1) h^{-n} .
$$

We can define the following relative determinants and their logarithms for $z \in R$ :

$$
\begin{gathered}
\mathcal{D}_{P}(z)=\ln \left|\operatorname{det}(P-z)\left(P_{\varepsilon}^{\mathrm{ext}}-z\right)^{-1}\right|, \\
\mathcal{D}_{P_{\varepsilon}}(z)=\ln \left|\operatorname{det}\left(P_{\varepsilon}-z\right)\left(P_{\varepsilon}^{\mathrm{ext}}-z\right)^{-1}\right|, \\
\mathcal{D}_{P_{\varepsilon, \delta}}(z)=\ln \left|\operatorname{det}\left(P_{\varepsilon, \delta}-z\right)\left(P_{\varepsilon}^{\mathrm{ext}}-z\right)^{-1}\right| .
\end{gathered}
$$

Here $P_{\varepsilon, \delta}=P_{\varepsilon, A, B, \delta}$ is given in Section 10. We derive some upper bounds:

Write

$$
(P-z)\left(P_{\varepsilon}^{\mathrm{ext}}-z\right)^{-1}=1-\left(P_{\varepsilon}^{\mathrm{ext}}-P\right)\left(P_{\varepsilon}^{\mathrm{ext}}-z\right)^{-1} .
$$

The last term is of trace class, so $\mathcal{D}_{P}$ is well defined. More precisely,

$$
\left\|\left(P_{\varepsilon}-P\right)\left(P_{\varepsilon}^{\mathrm{ext}}-z\right)^{-1}\right\|_{\mathrm{tr}} \leq\left\|P_{\varepsilon}^{\mathrm{ext}}-P\right\|_{\mathrm{tr}}\left\|\left(P_{\varepsilon}^{\mathrm{ext}}-z\right)^{-1}\right\| \leq \mathcal{O}\left(\varepsilon^{-1}\right) h^{-n},
$$

$\left({ }^{7}\right)$ Notice that if we put $\varepsilon_{\text {new }}=\frac{\varepsilon}{\widetilde{C}}$ for $\widetilde{C}$ fixed large enough, then the set $R$ will contain a rectangle of the form $]-\mathcal{O}\left(\varepsilon_{\text {new }}\right), \varepsilon_{\text {new }}[+i]-\varepsilon_{\text {new }}, \varepsilon_{\text {new }}[$ and we recover the scales in Theorem 1.1 with $\varepsilon$ replaced by the rescaled $\varepsilon_{\text {new }}$. 
where the norms and trace class norms are the ones for operators in $H\left(\Lambda_{t G}\right)$. Since in general (see [10]),

$$
|\operatorname{det}(1+K)| \leq \exp \|K\|_{\text {tr }}
$$

we conclude that

$$
\mathcal{D}_{P}(z) \leq \mathcal{O}(1) \varepsilon^{-1} h^{-n}
$$

Similarly,

$$
\left\|P_{\varepsilon}^{\mathrm{ext}}-P_{\varepsilon}\right\|_{\mathrm{tr}},\left\|P_{\varepsilon}^{\mathrm{ext}}-P_{\varepsilon, \delta}\right\|_{\mathrm{tr}}=\mathcal{O}\left(h^{-n}\right),
$$

so $\mathcal{P}_{P_{\varepsilon}}(z), \mathcal{D}_{P_{\varepsilon}, \delta}(z)$ are well defined and satisfy

$$
\mathcal{P}_{P_{\varepsilon}}(z), \mathcal{D}_{P_{\varepsilon, \delta}}(z) \leq \mathcal{O}(1) \varepsilon^{-1} h^{-n}
$$

Next, look at

$$
\begin{aligned}
\mathcal{D}_{P}(z)-\mathcal{D}_{P_{\varepsilon}}(z)=\ln \left|\operatorname{det}(P-z)\left(P_{\varepsilon}-z\right)^{-1}\right| & \\
& =\ln \left|\operatorname{det}\left(1-\left(P_{\varepsilon}-P\right)\left(P_{\varepsilon}-z\right)^{-1}\right)\right|
\end{aligned}
$$

which is well defined away from $\sigma\left(P_{\varepsilon}\right)$ and bounded from above by

$$
\begin{aligned}
\left.\|\left(P_{\varepsilon}-P\right)\left(P_{\varepsilon}-z\right)^{-1}\right) \|_{\mathrm{tr}} & \leq\left\|P_{\varepsilon}-P\right\|_{\mathrm{tr}}\left\|\left(P_{\varepsilon}-z\right)^{-1}\right\| \\
& \leq \mathcal{O}(1) \varepsilon^{n+1} h^{-n}\left\|\left(P_{\varepsilon}-z\right)^{-1}\right\| .
\end{aligned}
$$

We know from (9.19), that

$$
\left\|\left(P_{\varepsilon}-z\right)^{-1}\right\| \leq \frac{\mathcal{O}(1)}{\varepsilon \delta}, \text { for } z \in R \quad \text { with }|\Im z|>\delta \varepsilon .
$$

This is uniform for $0<\varepsilon \leq \varepsilon(\delta) \ll 1,0<h \leq h(\delta, \varepsilon)$. From this and (11.11), (11.10), we get the upper bound,

$$
\mathcal{D}_{P}(z)-\mathcal{D}_{P_{\varepsilon}}(z) \leq \mathcal{O}(1) \frac{1}{\delta}\left(\frac{\varepsilon}{h}\right)^{n}, \text { for } z \in R \text { with }|\Im z|>\delta \varepsilon .
$$

We also have lower bounds in a smaller part of $R$. From Proposition 10.1 we know that

$$
\left\|(P-z)^{-1}\right\| \leq \frac{\mathcal{O}(1)}{\delta \varepsilon}
$$

for

$$
z \in R \quad \text { with }|\Im z|>\delta \varepsilon \text { and } \Re z<-\frac{\varepsilon}{\mathcal{O}(1)}
$$


where the upper bound on $\Re z$ is the same as in (10.1). Exchanging $P$ and $P_{\varepsilon}$ in (11.10),

$$
\mathcal{D}_{P_{\varepsilon}}(z)-\mathcal{D}_{P}(z)=\ln \mid \operatorname{det}\left(1-\left(P-P_{\varepsilon}\right)(P-z)^{-1} \mid,\right.
$$

we then get, for $z$ in the subset (11.15),

$$
\mathcal{D}_{P}(z)-\mathcal{D}_{P_{\varepsilon}}(z) \geq-\mathcal{O}(1) \frac{1}{\delta}\left(\frac{\varepsilon}{h}\right)^{n} .
$$

We shall apply Jensen's formula and related estimates, following [15, Section 5]. Assume for simplicity that we have $-\frac{\varepsilon}{\mathcal{O}(1)}=-\frac{\varepsilon}{2}$ in (11.15). Let

$$
z_{0}=-\varepsilon-i \frac{\varepsilon}{2 C_{1}}
$$

where $C_{1}$ is the constant in (11.1), so that (11.17) holds for $z=z_{0}$. (The following can also be carried out in the upper half-plane with $z_{0}=-\varepsilon+i \frac{\varepsilon}{2 C_{1}}$.) It will be convenient to work in the rescaled variable $\tilde{z}$ with $z=\varepsilon \tilde{z}$, so that

$$
\tilde{z}_{0}=-1-i \frac{1}{2 C_{1}}
$$

and we put $\widetilde{\mathcal{D}}_{P}(\tilde{z})=\mathcal{D}_{P}(z)$ and similarly for the other $\mathcal{D}_{\left.P_{(} \ldots\right)}$. Let $r_{0}=\varepsilon \tilde{r}_{0}$ be the largest number such that

$$
D\left(z_{0}, r_{0}\right) \subset R_{\delta}:=\{z \in R ; \Im z<-\delta \varepsilon\}
$$

(More explicitly, $r_{0}=\left(\frac{1}{2 C_{1}}-\delta\right) \varepsilon$.) Consider the holomorphic function $\tilde{f}(\tilde{z})=f(z)$,

$$
f(z)=\operatorname{det}\left((P-z)\left(P_{\varepsilon}-z\right)^{-1}\right)
$$

for $z \in D\left(z_{0}, r_{0}\right)$ (corresponding to $\left.\tilde{z} \in D\left(\tilde{z}_{0}, \tilde{r}_{0}\right)\right)$. By (11.13), we have

$$
|f(z)| \leq \exp \left(\mathcal{O}(1) \frac{1}{\delta}\left(\frac{\varepsilon}{h}\right)^{n}\right), \quad z \in D\left(z_{0}, r_{0}\right) .
$$

Moreover,

$$
\left|f\left(z_{0}\right)\right| \geq \exp \left(-\mathcal{O}(1) \frac{1}{\delta}\left(\frac{\varepsilon}{h}\right)^{n}\right)
$$

since (11.17) holds for $z=z_{0}$. From Jensen's formula it follows that the number of zeros of $\tilde{f}$ in $D\left(\tilde{z}_{0},(1-\theta) \tilde{r}_{0}\right)$ is $\leq \mathcal{O}_{\delta}(1) \varepsilon^{n} h^{-n}$, if $\left.\theta \in\right] 0,1$ [ is any fixed constant. Equivalently, $f$ has $\leq \mathcal{O}_{\delta}(1) \varepsilon^{n} h^{-n}$ zeros in $D\left(z_{0},(1-\theta) r_{0}\right)$. 
Let $z_{j}=\varepsilon \tilde{z}_{j}, j=1,2, \ldots, N$ be the zeros of $f$ in $D\left(z_{0},(1-\theta) r_{0}\right)$, repeated according to their multiplicity, and put

$$
D_{w}(z ; h)=\prod_{j=1}^{N}\left(\tilde{z}-\tilde{z}_{j}\right), \quad z=\varepsilon \tilde{z} .
$$

Repeating the (standard) arguments in [15], we see that

$$
\left|D_{w}(z ; h)\right| \leq \exp \left(\mathcal{O}_{\delta}(1) \varepsilon^{n} h^{-n}\right) \quad \text { in } D\left(z_{0},(1-\theta) r_{0}\right)
$$

and that for any interval $I \in\left[0,(1-\theta) \tilde{r}_{0}\left[\right.\right.$ of length $|I|>0$ there exists $\tilde{r}_{1} \in I$ such that

$$
\left|D_{w}(z ; h)\right| \geq \exp \left(-\mathcal{O}_{\delta,|I|}(1)\left(\frac{\varepsilon}{h}\right)^{n}\right) \quad \text { when }\left|z-z_{0}\right|=r_{1}:=\varepsilon \tilde{r}_{1}
$$

Next, write

$$
f(z)=e^{G(z)} D_{w}(z ; h),
$$

with $G$ holomorphic in $D\left(z_{0},(1-\theta) r_{0}\right)$. Using the above bounds and Harnack's inequality (as in [15, Section 5]) we get

$$
|G(z)| \leq \mathcal{O}_{\delta}(1) \varepsilon^{n} h^{-n}, \quad z \in D\left(z_{0},(1-\theta)^{2} r_{0}\right)
$$

and for any interval $I \in\left[0,(1-\theta)^{2} \tilde{r}_{0}\right.$ [ of length $|I|>0$ there exists $\tilde{r}_{1} \in I$ such that

$$
|f(z)| \geq \exp \left(-\mathcal{O}_{\delta,|I|}(1) \varepsilon^{n} h^{-n}\right) \quad \text { when }\left|z-z_{0}\right|=r_{1}:=\varepsilon \tilde{r}_{1} .
$$

In other words,

$$
\mathcal{D}_{P}-\mathcal{D}_{P_{\varepsilon}} \geq-\mathcal{O}_{\delta,|I|}(1) \varepsilon^{n} h^{-n}
$$

for $z$ as in (11.28). Here we can take $|I|=(1-\theta)^{2} \tilde{r}_{0}-(1-\theta)^{3} \tilde{r}_{0}$ and find a corresponding $r_{1}$ with $(1-\theta)^{3} r_{0} \leq r_{1}<(1-\theta)^{2} r_{0}$.

The argument can now be repeated, by replacing $z_{0}$ by any new point on $\partial D\left(z_{0}, r_{1}\right) \ldots$ In this way, we continue until we have covered $R_{\delta} \backslash\left(\partial R_{\delta}+D(0, \delta \varepsilon)\right)$ with $\mathcal{O}_{\delta}(1)$ discs, and recalling that the zeros of $f$ in (11.21) are the resonances of $P$ in $R_{\delta}$, we get the following result:

Proposition 11.1. Define $R_{\delta}$ as in (11.20), (11.1). Then

(A) The number of resonances in $R_{\delta} \backslash\left(\partial R_{\delta}+D(0, \varepsilon \delta)\right)$ is $\leq \mathcal{O}_{\delta}(1) \varepsilon^{n} h^{-n}$ with the usual convention that $0<\varepsilon \leq \varepsilon(\delta), 0<h \leq h(\delta, \varepsilon)$.

(B) For all $a, b$ with $0<a<b<1$ independent of $\varepsilon, \delta$ and all segments $J \subset I \subset R_{\delta} \backslash$ $\left(\partial R_{\delta}+D(0, \varepsilon \delta)\right)$ of lengths $|J|=a \varepsilon,|I|=b \varepsilon$, there exists $z \in J$ such that

$$
\mathcal{D}_{P}(z)-\mathcal{D}_{P_{\varepsilon}}(z) \geq-\mathcal{O}_{a, b, \delta}(1) \varepsilon^{n} h^{-n} .
$$


Notice that Proposition 11.1 remains valid if we replace $R_{\delta}$, defined in (11.20), with

$$
R_{\delta}^{+}=\{z \in R ; \Im z>\varepsilon \delta\}
$$

Then $(A)$ holds trivially since there are no resonances in the upper half-plane.

We recall the bounds $(11.13),(11.17)$. In order to simplify the notations, we assume that the proposition is valid in all of $R_{\delta}$ (and in $R_{\delta}^{+}$), as can be achieved by a slight dilation of the parameters.

In order to complete the proof of Theorem 1.1, we shall work with $\mathcal{D}_{P}(z)-$ $\mathcal{D}_{P_{\varepsilon, \delta}}(z)$, exploiting the fact that $\mathcal{D}_{P_{\varepsilon, \delta}}$ is harmonic in

$$
R_{\varepsilon, \delta}:=\left\{z \in R ;|\Im z|>\varepsilon \delta \text { or }|\Re z-A \varepsilon|<\frac{\varepsilon \delta}{4} \text { or }|\Re z-B \varepsilon|<\frac{\varepsilon \delta}{4}\right\} .
$$

See Figure 2. Here $P_{\varepsilon, \delta}=P_{\varepsilon, A, B, \delta}$ is discussed in Section 10 and we know from that discussion that

$$
\left\|P_{\varepsilon}-P_{\varepsilon, \delta}\right\| \leq \mathcal{O}(\varepsilon \delta), \quad\left\|P_{\varepsilon}-P_{\varepsilon, \delta}\right\|_{\mathrm{tr}} \leq \mathcal{O}(\varepsilon \delta) \frac{\varepsilon \delta}{h^{n}}
$$

For $z$ in the region $R_{\varepsilon, \delta}$ we have

$$
\begin{aligned}
\mathcal{D}_{P_{\varepsilon}}(z)-\mathcal{D}_{P_{\varepsilon, \delta}}(z) & =\ln \left|\operatorname{det}\left(P_{\varepsilon}-z\right)\left(P_{\varepsilon, \delta}-z\right)^{-1}\right| \\
& \leq\left\|\left(P_{\varepsilon}-P_{\varepsilon, \delta}\right)\left(P_{\varepsilon, \delta}-z\right)^{-1}\right\|_{\operatorname{tr}} \\
& \leq\left\|P_{\varepsilon}-P_{\varepsilon, \delta}\right\|_{\operatorname{tr}}\left\|\left(P_{\varepsilon, \delta}-z\right)^{-1}\right\| \\
& \leq \mathcal{O}(1) \frac{(\varepsilon \delta)^{2}}{h^{n}} \frac{1}{\varepsilon \delta} \\
& =\mathcal{O}(1) \frac{\varepsilon \delta}{h^{n}} .
\end{aligned}
$$

Here we also use (10.11) or rather its natural analogue for $P_{\varepsilon, A, B, \delta}$.

Since $\left\|P-P_{\varepsilon}\right\|_{\text {tr }} \leq \mathcal{O}(1) \varepsilon^{n+1} h^{-n} \leq \mathcal{O}(1)(\varepsilon \delta)^{2} h^{-n}$ when $0<\varepsilon \leq \varepsilon(\delta)$, we have

$$
\mathcal{D}_{P}-\mathcal{D}_{P_{\varepsilon, \delta}} \leq \mathcal{O}(\varepsilon \delta) h^{-n}
$$

in (11.32). Indeed, this follows from (11.33) after replacing $P_{\varepsilon}$ there with $P$.

On the smaller set

$$
R_{\delta}:=\{z \in R ;|\Im z|>\varepsilon \delta\}
$$

we have

$$
\left\|\left(P_{\varepsilon}-z\right)^{-1}\right\| \leq \frac{\mathcal{O}(1)}{\delta \varepsilon}
$$


and exchanging $P_{\varepsilon}$ and $P_{\varepsilon, \delta}$ in (11.33), we get $\mathcal{D}_{P_{\varepsilon}}-\mathcal{D}_{P_{\varepsilon, \delta}} \geq-\mathcal{O}(\varepsilon \delta) h^{-n}$, hence with (11.33):

$$
\left|\mathcal{D}_{P_{\varepsilon}}-\mathcal{D}_{P_{\varepsilon, \delta}}\right| \leq \mathcal{O}(1) \frac{\varepsilon \delta}{h^{n}}
$$

for $z$ in the set (11.35). This means that the estimates (11.13), (11.30) for $\mathcal{D}_{P}-$ $\mathcal{D}_{P_{\varepsilon}}$ carry over to $\mathcal{D}_{P}-\mathcal{D}_{P_{\varepsilon}, \delta}$, provided that we replace the remainder estimates $\mathcal{O}_{\ldots} .(1) \varepsilon^{n} h^{-n}$ by $\mathcal{O}(\varepsilon \delta) h^{-n}$ : For $z \in R_{\delta}$, we have statement $(B)$ in Proposition 11.1 with

$$
\mathcal{D}_{P}(z)-\mathcal{D}_{P_{\varepsilon, \delta}}(z) \geq-\mathcal{O}(\varepsilon \delta) h^{-n}
$$

instead of (11.30).

To get lower bounds in $A \varepsilon+]-\frac{\varepsilon \delta}{4}, \frac{\varepsilon \delta}{4}[+i]-\varepsilon \delta, \varepsilon \delta[$ (and similarly with $B$ instead of $A$, we can apply the above arguments for $\mathcal{D}_{P}-\mathcal{D}_{P_{\varepsilon}}$ in $R_{\delta}$ to $\mathcal{D}_{P}-\mathcal{D}_{P_{\varepsilon, \delta}}$ in $A \varepsilon+$ ]$-\frac{\varepsilon \delta}{4}, \frac{\varepsilon \delta}{4}[+i]-\varepsilon \delta, \varepsilon \delta\left[\right.$, now starting at the point $z_{0}=A \varepsilon+i 2 \varepsilon \delta$ and get:

\section{Proposition 11.2.}

(A) The number of resonances of $P$ in $A \varepsilon+]-\frac{\varepsilon \delta}{4}, \frac{\varepsilon \delta}{4}[+i]-\varepsilon \delta, \varepsilon \delta[($ i.e. the zeros of $\left.\operatorname{det}\left((P-z)\left(P_{\varepsilon, \delta}-z\right)^{-1}\right)\right)$ is $\leq \mathcal{O}(\varepsilon \delta) h^{-n}$.

(B) For all $a, b$ with $0<a<b<1$, independent of $\varepsilon, \delta$ and all segments $J \subset I \subset$ $A \varepsilon+]-\frac{\varepsilon \delta}{4}, \frac{\varepsilon \delta}{4}[+i]-\varepsilon \delta, \varepsilon \delta[$ of length $|J|=a \delta \varepsilon,|I|=b \delta \varepsilon$, there exist $z \in J$, such that

$$
\mathcal{D}_{P}(z)-\mathcal{D}_{P_{\varepsilon, \delta}}(z) \geq-\mathcal{O}_{a, b}(\varepsilon \delta) h^{-n}
$$

The same statements hold with $B$ instead of $A$.

\section{End of the proof}

We study the number of resonances in the rectangle

$$
\Gamma=] A \varepsilon, B \varepsilon[+i]-\frac{\varepsilon}{2 C_{1}}, \frac{\varepsilon}{2 C_{1}}[,
$$

where $A, B$ and $C_{1}$ are positive constants, (see (11.1) and Section 10). Set

$$
\gamma=\partial \Gamma
$$

Again it is convenient to scale: $z=\varepsilon \tilde{z}$ and use $\widetilde{\gamma}=\partial \widetilde{\Gamma}$ where

$$
\widetilde{\Gamma}=] A, B[+i]-\frac{1}{2 C_{1}}, \frac{1}{2 C_{1}}[.
$$


We shall apply Theorem 1.1 of [16], (see also [17, Theorem 12.1.1]). In the rescaled variable $\tilde{z}$, we choose the Lipschitz weight on $\widetilde{\gamma}$ :

$$
\tilde{d}(\tilde{z})=\frac{1}{C}\left(\delta+\frac{1}{2}|\Im(\tilde{z})|\right), \quad \text { with } C>1 \text { large enough, }
$$

satisfying (cf. [16, (1.1)-(1.5)]):

$$
|\tilde{d}(\tilde{z})-\tilde{d}(\widetilde{w})| \leq \frac{1}{2}|\tilde{z}-\widetilde{w}|, \quad \forall \tilde{z}, \widetilde{w} \in \widetilde{\gamma}
$$

Extend $\tilde{d}$ to all $\mathbb{C}$ by setting

$$
\tilde{d}(\tilde{z})=\inf _{\widetilde{w} \in \widetilde{\gamma}}\left(\tilde{d}(\widetilde{w})+\frac{1}{2}|\tilde{z}-\widetilde{w}|\right), \quad \forall \tilde{z} \in \mathbb{C} .
$$

This extended function is also Lipschitz of modulus at most $\frac{1}{2}$, such that $\tilde{d}(\tilde{z}) \geq$ $\frac{1}{2} \operatorname{dist}(\tilde{z}, \widetilde{\gamma})$ and

$$
|\tilde{z}-\widetilde{w}| \leq \tilde{d}(\widetilde{w}) \Longrightarrow \frac{\tilde{d}(\widetilde{w})}{2} \leq \tilde{d}(\tilde{z}) \leq \frac{3 \tilde{d}(\widetilde{w})}{2}
$$

Choose $\tilde{z}_{j}^{0} \in \widetilde{\gamma}, j=0,1, \ldots, N-1$ distributed along $\widetilde{\gamma}=\partial \widetilde{\Gamma}$ in the positively oriented sense such that

$$
\frac{\tilde{d}\left(\tilde{z}_{j}^{0}\right)}{k} \leq\left|\tilde{z}_{j+1}^{0}-\tilde{z}_{j}^{0}\right| \leq \frac{\tilde{d}\left(\tilde{z}_{j}^{0}\right)}{2}, \quad 0 \leq j \leq N-1
$$

with the convention that $j+1=0$ when $j=N-1$, and for some $k>2$. Define

$$
\varphi(z):=h^{n}\left(\mathcal{D}_{P_{\varepsilon, \delta}}(z)+C \varepsilon \delta\right)
$$

with $C>0$ large enough so that

$$
\mathcal{D}_{P}(z) \leq h^{-n} \varphi(z) \quad \text { in the set (11.32). }
$$

The $\tilde{z}_{j}^{0}$ in $\left.\{A, B\}+i\right]-\delta, \delta[$ are choosen according to $(B)$ in Proposition 11.2 so that (11.39) holds when $z=z_{j}^{0}:=\varepsilon \tilde{z}_{j}^{0}$. Hence

$$
\mathcal{D}_{P}\left(z_{j}^{0}\right) \geq h^{-n}\left(\varphi\left(z_{j}^{0}\right)-\varepsilon_{j}\right),
$$

where $\varepsilon_{j}>0$ is independent of $j$, of the form

$$
\varepsilon_{j}=C_{0} \varepsilon \delta
$$

with $C_{0}>0$ large enough. The $z_{j}^{0} \in \partial \Gamma$ with $\left|\Im z_{j}^{0}\right|>\varepsilon \delta$ are chosen according to $(B)$ in Proposition 11.1, for which we have (11.38) and hence (12.8).

Consider the points $\tilde{z}_{1}^{0}, \tilde{z}_{2}^{0}, \ldots, \tilde{z}_{K}^{0}$ on $\left.B+i\right] \delta, \frac{1}{2 C_{1}}$ [ (possibly after relabeling) ordered so that $\Im\left(\tilde{z}_{1}^{0}\right)<\Im\left(\tilde{z}_{2}^{0}\right)<\ldots<\Im\left(\tilde{z}_{K}^{0}\right)$. From (12.5) we see that $\Im\left(\tilde{z}_{j}^{0}\right)$ growths 
geometrically with $j$ and it follows that $K \leq \mathcal{O}(1)|\ln (\delta)|$. The same holds for the corresponding points on $B+i]-\frac{1}{2 C_{1}},-\delta[, A+i] \delta, \frac{1}{2 C_{1}}[$ and $A+i]-\frac{1}{2 C_{1}},-\delta[$. The total number of points $\tilde{z}_{j}^{0}$ on $\widetilde{\gamma}$ is $N=\mathcal{O}(1)|\ln (\delta)|$.

Notice that $\phi(z)$ is harmonic in $\bigcup_{j=0}^{N-1} D\left(z_{j}^{0}, r_{j}\right)$. Apply Theorem 1.1 in [16] (or [17, Theorem 12.1.1]) with $h$ there is replaced by $h^{n}$ : We get in view of (12.9),

$$
\begin{aligned}
\left|\#\left(\mathcal{D}_{P}^{-1}(0) \cap \Gamma\right)-\frac{1}{2 \pi h^{n}} \int_{\Gamma} \Delta\left(h^{n} \mathcal{D}_{P_{\varepsilon, \delta}}(z)\right) L(d z)\right| & \leq \mathcal{O}(1) h^{-n} N \varepsilon \delta \\
& =\mathcal{O}(1) h^{-n} \varepsilon \delta|\ln \delta|
\end{aligned}
$$

where we first work in the $\tilde{z}$-variable but notice that

$$
\int_{\frac{1}{\varepsilon} \Gamma} \Delta_{\tilde{z}}\left(\mathcal{D}_{P_{\varepsilon, \delta}}(\tilde{z})\right) L(d \tilde{z})=\int_{\Gamma} \Delta_{z}\left(\mathcal{D}_{P_{\varepsilon, \delta}}(z)\right) L(d z) .
$$

Here $\frac{1}{2 \pi} \int_{\Gamma} \Delta\left(h^{n} \mathcal{D}_{P_{\varepsilon, \delta}}(z) L(d z)\right.$ is equal to the number of zeros in $\Gamma$ of $\mathcal{D}_{P_{\varepsilon, \delta}}$ or equivalently the number of resonances in $\Gamma$ of $P_{\varepsilon, \delta}$. By (10.12) this number is equal to $(2 \pi h)^{-n}(\omega(\varepsilon B)-\omega(\varepsilon A))+\mathcal{O}(\delta|\ln \delta| \varepsilon) h^{-n}$. This gives $(B)$ in Theorem 1.1 (with $a=\varepsilon A$ and $b=\varepsilon B$ ), since we already have the part (A) which follows from Proposition 11.1, (A).

\section{A. Review of [11] and adaptation to the dilated situation}

In this appendix we recall very briefly some basic microlocal tools developed in [11] for the study of semiclassical resonances and adapt them to our situation. To some extent, we shall follow the review in [12, Section 5].

\section{A.1. Order functions and symbols:}

Let $R, r \in C^{\infty}\left(\mathbb{R}^{n} ;\right] 0,+\infty[)$, satisfy for all $\alpha \in \mathbb{N}^{n}$ :

$$
\begin{gathered}
\partial_{x}^{\alpha} R(x)=\mathcal{O}(1) R(x)^{1-|\alpha|}, \\
\partial_{x}^{\alpha} r(x)=\mathcal{O}(1) r(x) R(x)^{-|\alpha|} .
\end{gathered}
$$

Define $\tilde{r}(x, \xi) \in C^{\infty}\left(\mathbb{R}^{2 n} ;\right] 0,+\infty[)$ by

$$
\tilde{r}(x, \xi)=\left(r(x)^{2}+\xi^{2}\right)^{\frac{1}{2}} .
$$

Then

$$
\partial_{x}^{\alpha} \partial_{\xi}^{\beta} \tilde{r}(x, \xi)=\mathcal{O}(1) \tilde{r}(x, \xi)^{1-|\beta|} R(x)^{-|\alpha|} .
$$


We make the important assumption that

$$
r(x) \geq 1, \quad r(x) R(x) \geq 1 .
$$

The quantities $R, r, \tilde{r}$ are our basic scale functions. The functions $R$ and $\tilde{r}$ give the scale in $x$ and $\xi$, respectively.

Definition A.1. (Order functions)

(a) We say that $m \in C^{\infty}\left(\mathbb{R}^{2 n} ;\right] 0,+\infty[)$ is an order function if

$$
\partial_{x}^{\alpha} \partial_{\xi}^{\beta} m(x, \xi)=\mathcal{O}(1) m(x, \xi) R(x)^{-|\alpha|} \tilde{r}(x, \xi)^{-|\beta|},
$$

for all $\alpha, \beta \in \mathbb{N}^{n}$.

(b) A function $m=m(x) \in C^{\infty}\left(\mathbb{R}^{n} ;\right] 0,+\infty[)$, independent of $\xi$, is an order function if

$$
\partial_{x}^{\alpha} m(x)=\mathcal{O}(1) m(x) R(x)^{-|\alpha|},
$$

for all $\alpha \in \mathbb{N}^{n}$.

Any finite product $\left(m_{1}(x, \xi) \times \ldots \times m_{N}(x, \xi)\right)$ of order functions is an order function. We notice that $R, r, \tilde{r}$ are order functions.

Definition A.2. (Symbol classes) Let $m$ be an order function.

(a) We say that $a \in C^{\infty}\left(\mathbb{R}^{2 n}\right)$ is a symbol of order $m$ and write $a \in S\left(\mathbb{R}^{2 n} ; m\right)$, if for all $\alpha, \beta \in \mathbb{N}^{n}$,

$$
\partial_{x}^{\alpha} \partial_{\xi}^{\beta} a(x, \xi)=\mathcal{O}(1) m(x, \xi) R(x)^{-|\alpha|} \tilde{r}(x, \xi)^{-|\beta|} \quad \text { on } \mathbb{R}^{2 n} .
$$

(b) We write $a \in \dot{S}\left(\mathbb{R}^{2 n}, m\right)$ when $(A .7)$ holds for all $\alpha, \beta \in \mathbb{N}^{n}$ with $|\alpha|+|\beta|>0$.

(c) If $\mathcal{U}$ is open subset of $\mathbb{R}^{2 n}$ we define $S(\mathcal{U} ; m)$ similarly, replacing $\mathbb{R}^{2 n}$ by $\mathcal{U}$.

Sometimes $m, a, R, r$ depend on parameters. We then require (A.1), (A.2), (A.6), (A.7), to hold uniformly with respect to the parameters, if nothing else is specified.

For more details see [11, Chapter 1, pp. 7-15].

\section{A.2. IR-Lagrangian manifolds:}

Let $G \in \dot{S}\left(\mathbb{R}^{2 n} ; R \tilde{r}\right)$ be real-valued. Then the manifold

$$
\Lambda_{G}=\left\{(x, \xi) \in \mathbb{C}^{2 n} ; \Im(x, \xi)=H_{G}(\Re(x, \xi))\right\}
$$

is I-Lagrangian, i.e. Lagrangian in $\mathbb{C}^{2 n}$ for the real symplectic form $-\Im \sigma$, where $\sigma=\sum_{1 \leq j \leq n} d \xi_{j} \wedge d x_{j}$ is the complex symplectic form. 
The one-form $-\Im(\xi \cdot d x)_{\Lambda_{G}}$ is closed on $\mathbb{C}^{2 n}$ and hence exact for topological reasons. The primitive $H$ is unique up to a constant and we can choose

$$
H=-\Re \xi \cdot \Im x+G(\Re(x, \xi))=G(\Re(x, \xi))-\Re \xi \cdot G_{\xi}^{\prime}(\Re(x, \xi)) .
$$

If we also assume that $G$ is small in $\dot{S}(R \tilde{r})$, then $\Lambda_{G}$ is $\mathbf{R}$-symplectic, i.e. a symplectic sub-manifold of $\mathbb{C}^{2 n}$, equipped with the symplectic form $\Re \sigma$. In other words, $\sigma_{\left.\right|_{\Lambda_{G}}}$ is a (real) symplectic form on $\Lambda_{G}$ and we have the volume element

$$
d \alpha=\left.\frac{1}{n !}\left(\sigma^{\wedge n}\right)\right|_{\Lambda_{G}} .
$$

For more details see [11, Chapter 2, p. 16].

\section{A.3. FBI-transforms and weighted Hilbert spaces:}

Clearly (A.8) gives a parametrization

$$
\mathbb{R}^{2 n} \ni \rho \longmapsto \rho+i H_{G}(\rho)
$$

of $\Lambda_{G}$ and we can then define symbol spaces $S\left(\Lambda_{G} ; m\right)$ of functions on $\Lambda_{G}$ by pulling back functions and weights to $\mathbb{R}^{2 n}$. In particular, we define the scales $R$ and $\tilde{r}$ by this pull back.

Let $\lambda=\lambda(\alpha) \in S\left(\Lambda_{G} ; R^{-1} \tilde{r}\right)$ be positive, elliptic in the sense that $\lambda$ is nonvanishing and $\lambda^{-1} \in S\left(\Lambda_{G} ; R \tilde{r}^{-1}\right)$ and put

$$
\phi(\alpha, y)=\left(\alpha_{x}-y\right) \alpha_{\xi}+i \frac{\lambda(\alpha)}{2}\left(\alpha_{x}-y\right)^{2}
$$

with $\alpha=\left(\alpha_{x}, \alpha_{\xi}\right) \in \Lambda_{G}$ and $y \in \mathbb{C}^{n}$.

The amplitude will be a $\mathbb{C}^{n+1}$-valued smooth function $\mathbf{t}(\alpha, y ; h)$ on $\Lambda_{G} \times \mathbb{C}_{y}^{n}$ which is affine linear in $y$. When discussing symbol properties of such functions we restrict the attention to a region

$$
\left|y-\alpha_{x}\right|<\mathcal{O}(1) R\left(\alpha_{x}\right),
$$

and with this convention, we require that $\mathbf{t} \in h^{-\frac{3 n}{4}} S\left(\Lambda_{G} ; R^{-\frac{n}{4}} \tilde{r}^{\frac{n}{4}}\right)$ and that $\mathbf{t}$, $\partial_{y_{1}} \mathbf{t}, \ldots, \partial_{y_{n}} \mathbf{t}$ are maximally linearly independent in the sense that with $\mathbf{t}$ treated as a column vector,

$$
\left|\operatorname{det}\left(\mathbf{t} \partial_{y_{1}} \mathbf{t} \ldots \partial_{y_{n}} \mathbf{t}\right)\right| \asymp R^{-n}\left(h^{-\frac{3 n}{4}} R^{-\frac{n}{4}} \tilde{r}^{\frac{n}{4}}\right)^{n+1} .
$$

(Vector valued symbols appear naturally after substitution of variables in a Gaussian resolution of the identity, see [11, Section 4].) 
Notice that the determinant is independent of $y$. If $\mathcal{B}_{0}$ is the canonical basis in $\mathbb{C}^{n+1}$, we can choose, for all $\alpha \in \Lambda_{G}$ and $y \in \mathbb{C}^{n}$ satisfying (A.11),

$$
\mathbf{t}(\alpha, y ; h)=t_{0}(\alpha ; h)\left(\begin{array}{c}
1 \\
\frac{\alpha_{x_{1}}-y_{1}}{R\left(\alpha_{x}\right)} \\
\vdots \\
\frac{\alpha_{x_{n}}-y_{n}}{R\left(\alpha_{x}\right)}
\end{array}\right)_{\mathcal{B}_{0}},
$$

where $t_{0}(\bullet ; h) \in h^{-\frac{3 n}{4}} S\left(\Lambda_{G} ; R^{-\frac{n}{4}} \tilde{r}^{\frac{n}{4}}\right)$.

Let $\chi \in C_{0}^{\infty}\left(B\left(0, \frac{1}{C}\right)\right)$ be equal to one in $B\left(0, \frac{1}{2 C}\right)$, where $C>0$ is large enough. We define the FBI-transform

$$
T: \mathcal{D}^{\prime}\left(\mathbb{R}^{n}\right) \longrightarrow C^{\infty}\left(\Lambda_{G} ; \mathbb{C}^{n+1}\right)
$$

by

$$
T u(\alpha ; h)=\int e^{\frac{i}{h} \phi(\alpha, y)} \mathbf{t}(\alpha, y ; h) \chi_{\alpha}(y) u(y) d y,
$$

where $\chi_{\alpha}(y)=\chi\left(\frac{y_{1}-\Re \alpha_{x_{1}}}{R\left(\Re \alpha_{x}\right)}, \ldots, \frac{y_{n}-\Re \alpha_{x_{n}}}{R\left(\Re \alpha_{x}\right)}\right)$. Here the domain of integration is equal to $\mathbb{R}^{n}$ and the integral is defined as the bilinear scalar product of $u \in \mathcal{D}^{\prime}\left(\mathbb{R}^{n}\right)$ and a test function in $C_{0}^{\infty}\left(\mathbb{R}^{n}\right)$.

We assume from now on that $G$ belongs to $S\left(\mathbb{R}^{2 n} ; R \tilde{r}\right)$. We also assume:

$$
\left\{\begin{array}{l}
\text { There exist } g_{0}=g_{0}(x) \in S\left(\mathbb{R}^{2 n} ; R r\right), \text { such that } \\
G(x, \xi)-g_{0}(x) \text { has its support in a region where } \\
|\xi| \leq \mathcal{O}(r(x)) \text { and } G(x, \xi)-g_{0}(x) \text { is sufficiently } \\
\text { small in } S\left(\mathbb{R}^{2 n} ; R r\right) .
\end{array}\right.
$$

Notice that the order function $\tilde{r}$ is controlled by $r$ in the region $|\xi| \leq \mathcal{O}(r(x))$.

Let $H$ be given in (A.9). Then $H \in S\left(\Lambda_{G} ; R \tilde{r}\right)$. Using $T$ we shall define the function spaces $H\left(\Lambda_{G} ; m\right)$, essentially by requiring that

$$
T u \in L^{2}\left(\Lambda_{G} ; m^{2} e^{-\frac{2}{h} H} d \alpha\right) .
$$

Here, $m$ is an order function.

Let $G$ satisfy (A.15) and be sufficiently small in $S\left(\mathbb{R}^{2 n} ; R \tilde{r}\right)$, or more generally, assume (A.15). Define $H$ as in (A.9), let $m$ be an order function on $\Lambda_{G}$ and let $T$ be an associated FBI-transform as in (A.14). In [11, Proposition 4.4] it is shown that $T$ is injective on $C_{0}^{\infty}\left(\mathbb{R}^{n}\right)$ and also on more general Sobolev spaces with exponential weights, by the construction of an approximate left inverse of $T$ which works with exponentially small errors. See [11, Chapter 4, pp. 20-42] for accurate results. 
Definition A.3. (Sobolev spaces associated to the IR-manifolds) The set $H\left(\Lambda_{G} ; m\right)$ is the completion of $C_{0}^{\infty}\left(\mathbb{R}^{n}\right)$ for the norm

$$
\|u\|_{H\left(\Lambda_{G} ; m\right)}=\|T u\|_{L^{2}\left(\Lambda_{G} ; m^{2} e^{-\frac{2}{h} H} d \alpha\right)} .
$$

The following facts were established in [11, Chapter 5, pp. 43-54]:

- $H\left(\Lambda_{G} ; m\right)$ is a Hilbert space.

- If we modify the choice of $\lambda$ and $\mathbf{t}$ in the definition of $T$, we get the same space $H\left(\Lambda_{G} ; m\right)$ and the new norm is uniformly equivalent to the earlier one, when $h$ tends to 0 .

- If $G_{1} \leq G_{2}$ and $m_{1} \geq m_{2}$, then $H\left(\Lambda_{G_{1}} ; m_{1}\right) \subset H\left(\Lambda_{G_{2}} ; m_{2}\right)$, and the inclusion map is uniformly bounded.

- When $G=g(x)$ is independent of $\xi$ and $m=m_{0}(x)$, we get

$$
H\left(\Lambda_{G} ; m\right)=L^{2}\left(\mathbb{R}^{n} ; m_{0}^{2} e^{-\frac{2}{h} g(x)} d x\right)
$$

with uniform equivalence of norms. More generally, when $G=g(x)$ and $m(x, \xi)=$ $m_{0}(x)\left(\frac{\tilde{r}(x, \xi)}{r(x)}\right)^{N_{0}}$ with $N_{0} \in \mathbb{R}$, then $H\left(\Lambda_{G} ; m\right)$ is the naturally defined exponentially weighted Sobolev space.

\section{A.4. Schrödinger operator on $H\left(\Lambda_{G} ; m\right)$ :}

We now consider a Schrödinger operator

$$
P=-h^{2} \Delta+V(x), \quad x \in \mathbb{R}^{n},
$$

where $V$ is real-valued and

$$
V \in S\left(\mathbb{R}^{n} ; r^{2}\right)
$$

so that the symbol $p(x, \xi)=\xi^{2}+V(x)$ belongs to $S\left(\mathbb{R}^{2 n} ; \tilde{r}^{2}\right)$.

A basic element in the theory is that if $V$ extends holomorphically to a truncated sector $\Gamma_{C}$ as in (1.1), and the extension satisfies $|V(x)| \leq \mathcal{O}(1) r(\Re x)^{2}$, and if $G$ satisfies (A.15) with $G-g_{0}=0$ near the analytic singular support of $V$, then

$$
P: H\left(\Lambda_{G} ; \tilde{r}^{2}\right) \longrightarrow H\left(\Lambda_{G}\right)
$$

can be viewed as an $h$-pseudo-differential operator with leading symbol $p_{\left.\right|_{\Lambda_{G}}}$. For precise results, see $[11$, Chapter 6 , Théorèmes $6.8,6.8$ (corrigé), and the paragraph in the pages $77-78$ ]. 


\section{A.5. Dilations}

Let $\varepsilon_{0}$ be a positive small constant. For $\left.\left.\varepsilon \in\right] 0, \varepsilon_{0}\right]$, we introduce the basic scale functions:

$$
\begin{aligned}
& R_{\varepsilon}(x)=\left(\varepsilon+x^{2}\right)^{\frac{1}{2}}, \quad r_{\varepsilon}(x)=\frac{\left(\varepsilon+x^{2}\right)^{\frac{1}{2}}}{\left(1+x^{2}\right)^{\frac{1}{2}}} \\
& \text { and } \tilde{r}_{\varepsilon}(x, \xi)=\left(r_{\varepsilon}(x)^{2}+\xi^{2}\right)^{\frac{1}{2}} .
\end{aligned}
$$

As above, with these scales, we define the notion of order functions and symbols. To emphasize the dependence on the parameter $\varepsilon$, we add it in the notations as follows:

Definition A.4. ( $\varepsilon$-Order functions)

(a) We say that $m \in C^{\infty}\left(\mathbb{R}^{2 n} ;\right] 0,+\infty[)$ is an $\varepsilon$-order function if

$$
\partial_{x}^{\alpha} \partial_{\xi}^{\beta} m(x, \xi)=\mathcal{O}(1) m(x, \xi) R_{\varepsilon}(x)^{-|\alpha|} \tilde{r}_{\varepsilon}(x, \xi)^{-|\beta|},
$$

for all $\alpha, \beta \in \mathbb{N}^{n}$.

(b) A function $m=m(x) \in C^{\infty}\left(\mathbb{R}^{n} ;\right] 0,+\infty[)$, independent of $\xi$, is an $\varepsilon$-order function if

$$
\partial_{x}^{\alpha} m(x)=\mathcal{O}(1) m(x) R_{\varepsilon}(x)^{-|\alpha|},
$$

for all $\alpha \in \mathbb{N}^{n}$.

We require the estimates to be uniform in $\varepsilon$. In the special case when $\varepsilon$ is fixed $=1$, we get an order function in the sense of [11].

Definition A.5. ( $\varepsilon$-Symbol classes) Let $m$ be an $\varepsilon$-order function.

(a) We say that $a \in C^{\infty}\left(\mathbb{R}^{2 n}\right)$ is an $\varepsilon$-symbol of order $m$ and write $a \in$ $S_{\varepsilon}\left(\mathbb{R}^{2 n} ; m\right)$, if for all $\alpha, \beta \in \mathbb{N}^{n}$,

$$
\partial_{x}^{\alpha} \partial_{\xi}^{\beta} a(x, \xi)=\mathcal{O}(1) m(x, \xi) R_{\varepsilon}(x)^{-|\alpha|} \tilde{r}_{\varepsilon}(x, \xi)^{-|\beta|} \quad \text { on } \mathbb{R}^{2 n} .
$$

(b) We write $a \in \dot{S}_{\varepsilon}\left(\mathbb{R}^{2 n}, m\right)$ when $\left(\right.$ A.21) holds for all $\alpha, \beta \in \mathbb{N}^{n}$ with $|\alpha|+|\beta|>0$.

(c) If $\mathcal{U}$ is open subset of $\mathbb{R}^{2 n}$ we define $S_{\varepsilon}(\mathcal{U} ; m)$ similarly, replacing $\mathbb{R}^{2 n}$ by $\mathcal{U}$.

Now return to our Schrödinger operator $P$ as in Theorem 1.1. Let $G=G^{\varepsilon}$ or $G=G^{\frac{C \varepsilon}{C}}$ be the local escape functions in Propositions 3.1, 4.1, 5.1, 5.2. At the end of Section 5 we recalled how to extend $G$ to a global escape function $\widetilde{G}$ satisfying (5.22), (5.23) away from a fixed neighborhood of $(0,0)$. We now drop the tilde and denote by $G$ (or $G^{\varepsilon}, G^{\frac{C \varepsilon}{\tilde{C}}}$ ) this global escape function for which the above cited propositions hold near $(0,0)$, in addition to $(5.22),(5.23)$. We also have $G \in S_{\varepsilon}\left(\mathbb{R}^{2 n} ; R_{\varepsilon} \tilde{r}_{\varepsilon}\right)$ and we can arrange so that $|\xi| \leq \mathcal{O}(1) r_{\varepsilon}(x)$ for $(x, \xi) \in \operatorname{supp} G$. 
We wish to apply [11] to the operators $P, P_{\varepsilon}$ and $P_{\varepsilon}^{\text {ext }}$ given by $(6.1),(6.2)$ and (8.5) respectively with the scale functions $R=R_{\varepsilon}, r=r_{\varepsilon}, \tilde{r}_{\varepsilon}$ given by (A.19). Notice that these functions satisfy (A.1), (A.2), (A.4). However (A.5) does not hold near $x=0$ and we only have

$$
r_{\varepsilon}(x) \geq \sqrt{\varepsilon}, \quad R_{\varepsilon}(x) r_{\varepsilon}(x) \geq \varepsilon .
$$

In order to remedy for the failure of (A.5), we make the change of variables

$$
x=\mu \tilde{x} \quad \text { with } \mu=\sqrt{\varepsilon}
$$

and introduce a new semi-classical parameter, by requiring that

$$
h D_{x}=\mu \tilde{h} D_{\tilde{x}}, \quad \text { i.e., } \tilde{h}=\frac{h}{\mu^{2}} .
$$

Assuming from now on that

$$
\varepsilon \geq h^{\frac{1}{2}-\alpha_{0}}, \quad \text { for some fixed } \alpha_{0}>0,
$$

we see that $\tilde{h}$ tends to 0 when $h$ goes to 0 .

The corresponding dilation of a semi-classical operator $Q=q\left(x, h D_{x}\right)$ is $\widetilde{Q}=$ $q\left(\mu\left(\tilde{x}, \tilde{h} D_{\tilde{x}}\right)\right)$, whose semi-classical symbol (with respect to $\tilde{h}$ ) is given by

$$
\tilde{q}(\tilde{x}, \tilde{\xi})=q(\mu \tilde{x}, \mu \tilde{\xi}) .
$$

The balls $B\left(x ; R_{\varepsilon}(x)\right)$ and $B\left(\xi ; \tilde{r}_{\varepsilon}(x, \xi)\right)$ become $B\left(\tilde{x}, \frac{1}{\mu} R_{\varepsilon}(\mu \tilde{x})\right)$ and $B\left(\tilde{\xi}, \frac{1}{\mu} \tilde{r}_{\varepsilon}(\mu \tilde{x}, \mu \tilde{\xi})\right)$ respectively in the $\tilde{x}, \tilde{\xi}$ coordinates. It is then natural to put:

$$
\begin{gathered}
R_{\varepsilon, \mu}(\tilde{x})=\frac{1}{\mu} R_{\varepsilon}(\mu \tilde{x}), \quad r_{\varepsilon, \mu}(\tilde{x})=\frac{1}{\mu} r_{\varepsilon}(\mu \tilde{x}), \\
\tilde{r}_{\varepsilon, \mu}(\tilde{x}, \tilde{\xi})=\frac{1}{\mu} \tilde{r}_{\varepsilon}(\mu \tilde{x}, \mu \tilde{\xi})=\left(r_{\varepsilon, \mu}(\tilde{x})^{2}+\tilde{\xi}^{2}\right)^{\frac{1}{2}} .
\end{gathered}
$$

Recalling that $\mu=\sqrt{\varepsilon}$, we get more explicitly,

$$
R_{\varepsilon, \mu}(\tilde{x})=\left(1+\tilde{x}^{2}\right)^{\frac{1}{2}}, \quad r_{\varepsilon, \mu}(\tilde{x})=\frac{\left(1+\tilde{x}^{2}\right)^{\frac{1}{2}}}{\left(1+\varepsilon \tilde{x}^{2}\right)^{\frac{1}{2}}} .
$$

The rescaled functions satisfy (A.1), (A.2), ((A.4)) and (A.5):

$$
\begin{array}{cl}
\partial_{\tilde{x}}^{\alpha} R_{\varepsilon, \mu}=\mathcal{O}(1) R_{\varepsilon, \mu}^{1-|\alpha|}, & \partial_{\tilde{x}}^{\alpha} r_{\varepsilon, \mu}=\mathcal{O}(1) r_{\varepsilon, \mu} R_{\varepsilon, \mu}^{-|\alpha|} \\
r_{\varepsilon, \mu}(\tilde{x}) \geq 1 \quad \text { and } \quad & r_{\varepsilon, \mu}(\tilde{x}) R_{\varepsilon, \mu}(\tilde{x}) \geq 1 .
\end{array}
$$


Let $S_{\varepsilon, \mu}\left(\bullet ; m_{\varepsilon, \mu}\right)$ denote the symbol space $S(\bullet ; m)$, defined as above, but now with respect to the scales $r_{\varepsilon, \mu}, R_{\varepsilon, \mu}, \tilde{r}_{\varepsilon, \mu}$ (from now on $\mu$-scales for short), with $m_{\varepsilon, \mu}$ being an order function: $m_{\varepsilon, \mu} \in S_{\varepsilon, \mu}\left(\bullet ; m_{\varepsilon, \mu}\right)$.

If $m$ is an order function for the scales $r_{\varepsilon}, R_{\varepsilon}, \tilde{r}_{\varepsilon}$ (from now on $\varepsilon$-scales) and $a \in S_{\varepsilon}\left(\bullet ; m_{\varepsilon}\right)$ and if we define

$$
a_{\mu}(\tilde{x}, \tilde{\xi})=a(\mu(\tilde{x}, \tilde{\xi})), \quad m_{\varepsilon, \mu}(\tilde{x}, \tilde{\xi})=m_{\varepsilon}(\mu(\tilde{x}, \tilde{\xi}))
$$

then $m_{\mu}$ is an order function for the $\mu$-scales and $a_{\varepsilon, \mu} \in S_{\varepsilon, \mu}\left(m_{\mu}\right)$.

Consider an FBI-transform $T$ as in (A.10)-(A.14) and let $G, g_{0}$ be as in the corresponding discussion so that (A.15) holds with respect to the $\varepsilon$-scales. In (A.14) we make the change of variables,

$$
\alpha=\mu \widetilde{\alpha}, \quad y=\mu \tilde{y}
$$

and define $\tilde{u}(\tilde{y})$ by

$$
u(y)=\mu^{\frac{n}{2}} \tilde{u}(\tilde{y})
$$

so that the map

$$
\left\{\begin{aligned}
L^{2}\left(\mathbb{C}^{n} ; d y\right) & \longrightarrow L^{2}\left(\mathbb{C}^{n} ; d \tilde{y}\right) \\
u(\bullet) & \longmapsto \tilde{u}(\tilde{\bullet})
\end{aligned} \quad\right. \text { is unitary }
$$

We have $\frac{1}{h} \phi(\alpha, y)=\frac{1}{\tilde{h}} \phi_{\mu}(\widetilde{\alpha}, \tilde{y})$, where

$$
\phi_{\mu}(\widetilde{\alpha}, \tilde{y})=\frac{1}{\mu^{2}} \phi(\mu \widetilde{\alpha}, \mu \tilde{y})=\left(\widetilde{\alpha}_{x}-\tilde{y}\right) \cdot \widetilde{\alpha}_{\xi}+i \frac{\tilde{\lambda}(\widetilde{\alpha})}{2}\left(\widetilde{\alpha}_{x}-\tilde{y}\right)^{2}
$$

and $\tilde{\lambda}(\widetilde{\alpha})=\lambda\left(\frac{\widetilde{\alpha}}{\mu}\right) \in S_{\varepsilon, \mu}\left(\Lambda_{G_{\mu}} ; R_{\varepsilon, \mu} \tilde{r}_{\varepsilon, \mu}\right)$ is elliptic and positive. Here $\Lambda_{G_{\mu}}$ is the I-Lagrangian manifold associated to the escape function $G_{\mu}$ (in the $\mu$-scales) given by (A.39) below. We have

$$
T u(\alpha ; h)=T_{\mu} \tilde{u}(\widetilde{\alpha} ; \tilde{h})
$$

where

$$
\begin{gathered}
T_{\mu} \tilde{u}(\widetilde{\alpha} ; \tilde{h})=\int e^{\frac{i}{h} \tilde{\phi}(\alpha, y)} \mathbf{t}_{\mu}(\widetilde{\alpha}, \tilde{y} ; \tilde{h}) \chi_{\widetilde{\alpha}}(\tilde{y}) \tilde{u}(\tilde{y}) d \tilde{y} \\
\tilde{\chi}_{\widetilde{\alpha}}(\tilde{y})=\chi_{\alpha}(y)
\end{gathered}
$$

and

$$
\mathbf{t}_{\mu}(\widetilde{\alpha}, \tilde{y} ; \tilde{h})=\mu^{\frac{3 n}{2}} \mathbf{t}(\alpha, y ; h)=\mu^{\frac{3 n}{2}} \mathbf{t}\left(\mu \widetilde{\alpha}, \mu \tilde{y} ; \mu^{2} \tilde{h}\right)
$$


The function $\tilde{\chi}$ has the same cut-off properties in the $\mu$-scales as $\chi_{\alpha}$ in the $\varepsilon$-scales. Moreover, $\mathbf{t}_{\mu}$ is affine linear in $\tilde{y}$ and

$$
\mathbf{t}_{\mu} \in \tilde{h}^{-\frac{3 n}{4}} S_{\varepsilon, \mu}\left(\Lambda_{G_{\mu}} ; R_{\varepsilon, \mu}^{-\frac{n}{4}} \tilde{r}_{\varepsilon, \mu}^{\frac{n}{4}}\right) .
$$

From (A.12) we get by straight forward calculation,

$$
\left|\operatorname{det}\left(\mathbf{t}_{\mu} \partial_{\tilde{y}_{1}} \mathbf{t}_{\mu} \ldots \partial_{\tilde{y}_{n}} \mathbf{t}_{\mu}\right)\right| \asymp R_{\varepsilon, \mu}^{-n}\left(\tilde{h}^{-\frac{3 n}{4}} R_{\varepsilon, \mu}^{-\frac{n}{4}} \tilde{r}_{\varepsilon, \mu}^{\frac{n}{4}}\right)^{n+1},
$$

which is analogous to (A.12).

Let $G \in S\left(\mathbb{R}^{2 n} ; R_{\varepsilon} \tilde{r}_{\varepsilon}\right)$ be real-valued. Define $G_{\mu}$ by

$$
\frac{1}{h} G(\alpha)=\frac{1}{\tilde{h}} G_{\mu}(\widetilde{\alpha}) \text {, i.e. } G_{\mu}(\widetilde{\alpha})=\frac{1}{\mu^{2}} G\left(\frac{\widetilde{\alpha}}{\mu}\right) .
$$

Then

$$
G_{\mu} \in \mu^{-2} S_{\varepsilon, \mu}\left(\mathbb{R}^{2 n} ;\left(\mu R_{\varepsilon, \mu}\right)\left(\mu \tilde{r}_{\varepsilon, \mu}\right)\right)=S_{\varepsilon, \mu}\left(\mathbb{R}^{2 n} ; R_{\varepsilon, \mu} \tilde{r}_{\varepsilon, \mu}\right) .
$$

If (A.15) holds for the $\varepsilon$-scales, then it also holds for $G_{\mu}, g_{0, \mu}$ for the $\mu$-scales with $g_{0, \mu}\left(\widetilde{\alpha}_{x}\right)=\mu^{-2} g_{0}\left(\mu \widetilde{\alpha}_{x}\right)$.

With $H$ as in (A.9) we get by straight forward calculation,

$$
H(\alpha)=\mu^{2} H_{\mu}(\widetilde{\alpha})
$$

where

$$
H_{\mu}(\widetilde{\alpha})=G_{\mu}(\Re \widetilde{\alpha})-\Re \widetilde{\alpha}_{\xi} \cdot \partial_{\tilde{\xi}} G_{\mu}(\Re \widetilde{\alpha}) .
$$

Since the weights $R_{\varepsilon, \mu}, r_{\varepsilon, \mu}, \tilde{r}_{\varepsilon, \mu}$ satisfy (A.1), (A.2), (A.4) and (A.5) we can define the Sobolev spaces $\Lambda_{G_{\mu}}, H\left(\Lambda_{G_{\mu}} ; m_{\varepsilon, \mu}\right)$, associated to the IR-manifolds as in Definition A.3. In view of (A.33) this allows us to define the spaces $H\left(\Lambda_{G} ; m\right)$ for the scales $r_{\varepsilon}, R_{\varepsilon}, \tilde{r}_{\varepsilon}$ :

We say that $u \in H\left(\Lambda_{G} ; m_{\varepsilon}\right)$ if $\tilde{u} \in H\left(\Lambda_{G_{\mu}} ; m_{\varepsilon, \mu}\right)$ with $m \longleftrightarrow m_{\varepsilon, \mu}, u \longleftrightarrow \tilde{u}$ and $G \longleftrightarrow G_{\mu}$ related as above, see (A.31), (A.32) and (A.39).

For $u \in H\left(\Lambda_{G} ; m_{\varepsilon}\right)$ we choose the norm

$$
\|u\|_{H\left(\Lambda_{G} ; m_{\varepsilon}\right)}=\|T u\|_{L^{2}\left(\Lambda_{G} ; m_{\varepsilon}^{2} e^{-\frac{2}{h} H} d \alpha\right)} .
$$

Defining similarly

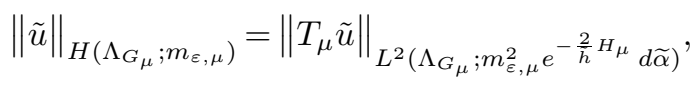

we find,

$$
\|u\|_{H\left(\Lambda_{G} ; m_{\varepsilon}\right)}=\mu^{n}\|\tilde{u}\|_{H\left(\Lambda_{G \mu} ; m_{\varepsilon, \mu}\right)},
$$


since $d \alpha=\mu^{2 n} d \widetilde{\alpha}$ and the relationships between the different involved quantities (See (A.31), (A.32), (A.39) and (A.40)).

In conclusion, the changes of variables above allow us to replace the scales $r_{\varepsilon}$, $R_{\varepsilon}, \tilde{r}_{\varepsilon}$ that do not satisfy (A.5) by the scales $r_{\varepsilon, \mu}, R_{\varepsilon, \mu}, \tilde{r}_{\varepsilon, \mu}$ that do so, and we can then apply the theory of [11].

\section{B. Volume functions}

Recall that $\omega(E)$ is defined by $(1.16)$ or (1.17) when $E \leq 0$. The saddle point being $x=0$, we choose coordinates as in the beginning of Section 3. We extend $\omega(E)$ to small positive values of $E$ by replacing $\mathscr{U}_{0}$ in (1.16) or (1.17) with $\widetilde{\mathscr{U}_{0}}:=$ $\left\{x \in \operatorname{neigh}\left(\overline{\mathscr{U}}_{0}, \mathbb{R}^{n}\right) ; x_{n} \leq 0\right.$ when $\left.x \in \operatorname{neigh}\left(0, \mathbb{R}^{n}\right)\right\}$. Then (1.18) remains valid again with $\mathscr{U}_{0}$ replaced by $\widetilde{\mathscr{U}_{0}}$ and this gives a nice $C^{1}$-extension of $\omega(E)$ to $E \in \operatorname{neigh}(0, \mathbb{R})$. The volume $\omega_{\varepsilon}(E)$ in $(7.5)$, can be written

$$
\omega_{\varepsilon}(E)=\int_{\widetilde{\mathscr{U}_{0}}}\left(\int_{p_{\varepsilon}(x, \xi) \leq E} d \xi\right) d x
$$

Recall that $p_{\varepsilon}$ is given by (4.8), (4.3). Assume for simplicity that $\chi(x, 0) \geq \chi(x, \xi)$ for all $\xi \in \mathbb{R}^{n}$. Then, since

$$
p_{\varepsilon}(x, \xi)=\xi^{2}+V(x)+\chi_{\varepsilon}(x, \xi)
$$

where $\chi_{\varepsilon}$ is given by $(4.3)$, we have

$$
\xi^{2}+V(x) \leq p_{\varepsilon}(x, \xi) \leq \xi^{2}+V(x)+\chi_{\varepsilon}(x, 0)
$$

and it follows that

$$
C_{n} \int_{\widetilde{\mathscr{U}}_{0}}\left(E-\left(V(x)+\chi_{\varepsilon}(x, 0)\right)\right)_{+}^{\frac{n}{2}} d x \leq \omega_{\varepsilon}(E) \leq C_{n} \int_{\widetilde{\mathscr{U}}_{0}}(E-V(x))_{+}^{\frac{n}{2}} d x
$$

where $C_{n}=\operatorname{vol}\left(B_{\mathbb{R}^{n}}(0,1)\right)=\frac{\pi^{\frac{n}{2}}}{\Gamma\left(\frac{n}{2}+1\right)}$, and the last member is $\omega(E)$ by (1.17). Using that $[0,1] \ni t \longmapsto\left(E-\left(V(x)+t \chi_{\varepsilon}(x, 0)\right)\right)_{+}^{\frac{n}{2}}$ is a convex function, we get

$$
(E-V(x))_{+}^{\frac{n}{2}}-\left(E-\left(V(x)+\chi_{\varepsilon}(x, 0)\right)\right)_{+}^{\frac{n}{2}} \leq \frac{n}{2}(E-V(x))_{+}^{\frac{n}{2}-1} \chi_{\varepsilon}(x, 0)
$$


so by (B.3):

$$
\begin{aligned}
0 \leq \omega(E)-\omega_{\varepsilon}(E) & \leq \frac{\pi^{\frac{n}{2}}}{\Gamma\left(\frac{n}{2}\right)} \int_{\widetilde{\mathscr{U}_{0}}}(E-V(x))_{+}^{\frac{n}{2}-1} \chi_{\varepsilon}(x, 0) d x \\
& \leq \mathcal{O}(1) \int_{\widetilde{\mathscr{U}_{0}}} \chi_{\varepsilon}(x, 0) d x \\
& \leq \mathcal{O}(\varepsilon) \varepsilon^{\frac{n}{2}} \\
& \leq \mathcal{O}(1) \varepsilon^{2} .
\end{aligned}
$$

\section{References}

1. Bony, J.-F., Fujié, S., Ramond, T. and Zerzeri, M., WKB Solutions Near an Unstable Equilibrium and Applications, in Nonlinear Physical Systems - Spectral Analysis, Stability and Bifurcations, pp. 15-39, ISTE, Wiley, 2014.

2. Combes, J.-M., Duclos, P., Klein, M. and Seiler, R., The shape resonance, Commun. Math. Phys. 110 (1987), 215-236.

3. Datchev, K. and Jin, L., Exponential lower resolvent bounds far away from trapped sets, J. Spectr. Theory 10 (1987), 617-649. 2020.

4. Dimassi, M. and SJöstrand, J., Spectral asymptotics in the semi-classical limit, Cambridge University Press, Cambridge, 1999.

5. Duyckaerts, T., Grigis, A. and Martinez, A., Resonance widths for general Helmholtz resonators with straight neck, Duke Math. J. 165 (2016), 27932810 .

6. Dyatlov, S. and Zworski, M., Mathematical Theory of Scattering Resonances, Graduate Studies in Mathematics 200, Am. Math. Soc., Providence, 2019.

7. Fujié, S., Lahmar-Benbernou, A. and Martinez, A., Width of shape resonances for non globally analytic potentials, J. Math. Soc. Jpn. 63 (2011), 1-78.

8. FujilÉ, S. and RAmond, T., Matrice de scattering et résonances associeées à une orbite hétérocline, Ann. IHP, Phys. Théor. 69 (1998), 31-82.

9. GÉrARd, C. and SJöstrand, J., Semiclassical resonances generated by a closed trajectory of hyperbolic type, Commun. Math. Phys. 108 (1987), 391-421.

10. Gohberg, I.-C. and Krein, M.-G., Introduction to the theory of linear non-selfadjoint operators, Translations of mathematical monographs 18, Am. Math. Soc., Providence, R.I., 1969.

11. Helffer, B. and Sjöstrand, J., Résonances en limite semiclassique, Bull. de la SMF. 114(3), Mémoire 24/25, 1986.

12. Hitrik, M., Mantile, A. and Sjöstrand, J., Adiabatic Evolution and shape resonances. Preprint, 2017. arXiv:1711.07583.

13. Martinez, A., Resonance free domains for non globally analytic potentials, Ann. Henri Poincaré 3 (2002), 739-756.

14. Nakamura, S., Stefanov, P. and Zworski, M., Resonance expansions of propagators in the presence of potential barriers, J. Funct. Anal. 205 (2003), 180-205.

15. Sjöstrand, J., Resonances for bottles and trace formulae, Math. Nachr. 221 (2001), 95-149. 
16. Sjöstrand, J., Counting zeros of holomorphic functions of exponential growth, $J$. Pseudo-Differ. Oper. Appl. 1 (2010), 75-100.

17. Sjöstrand, J., Non-self-adjoint differential operators, spectral asymptotics and random perturbations, in Pseudo-Differential Operators Theory and Applications 14, Birkhäuser, Basel, 2019.

Johannes Sjöstrand

Institut de Mathématiques de Bourgogne, UMR5584 CNRS

Université de Bourgogne Franche-Comté

Dijon F-2100

France

johannes.sjostrand@u-bourgogne.fr
Maher Zerzeri

Laboratoire Analyse, Géométrie et Applications, UMR7539 CNRS

Université Sorbonne Paris-Nord 99, avenue J.-B. Clément

Villetaneuse F-93430

France

zerzeri@math.univ-paris13.fr

Received April 10, 2020

in revised form December 10, 2020 